Network Working Group

Request for Comments: 2273

Obsoletes: 2263

Category: Standards Track

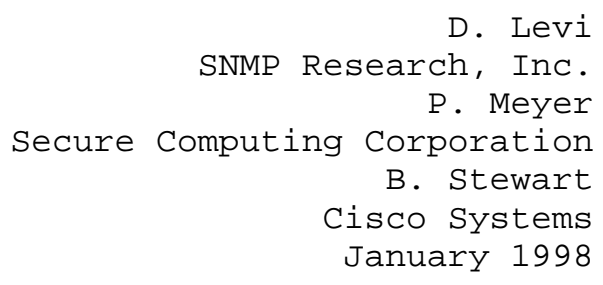

SNMPv3 Applications

Status of this Memo

This document specifies an Internet standards track protocol for the Internet community, and requests discussion and suggestions for improvements. Please refer to the current edition of the "Internet Official Protocol Standards" (STD 1) for the standardization state and status of this protocol. Distribution of this memo is unlimited.

Copyright Notice

Copyright (C) The Internet Society (1998). All Rights Reserved.

IANA Note

Due to a clerical error in the assignment of the snmpModules in this memo, this RFC provides the corrected number assignments for this protocol. This memo obsoletes RFC 2263.

Abstract

This memo describes five types of SNMP applications which make use of an SNMP engine as described in [RFC2271]. The types of application described are Command Generators, Command Responders, Notification Originators, Notification Receivers, and Proxy Forwarders.

This memo also defines MIB modules for specifying targets of management operations, for notification filtering, and for proxy forwarding.

Table of Contents

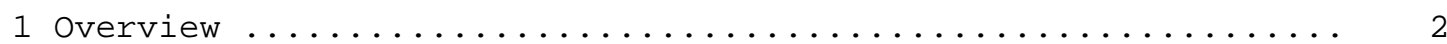

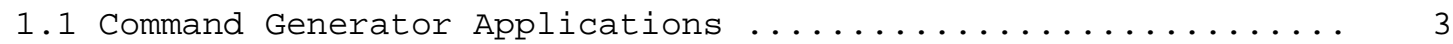

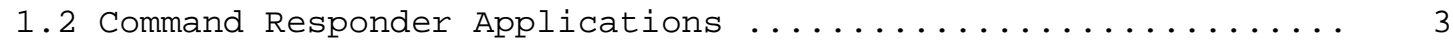

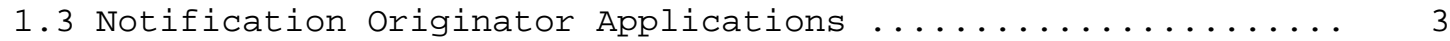

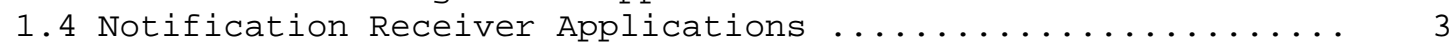

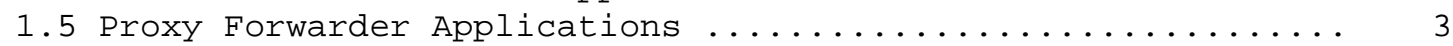

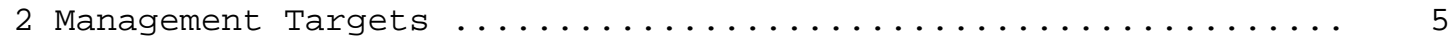




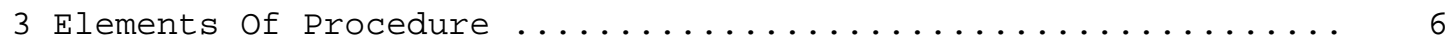

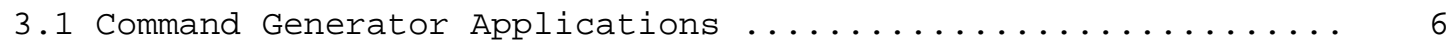

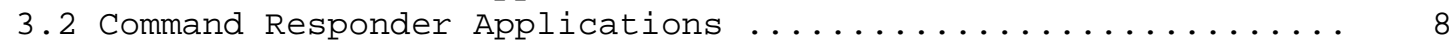

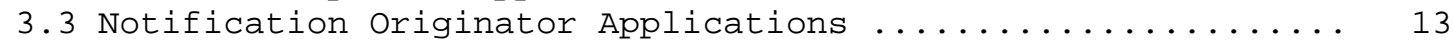

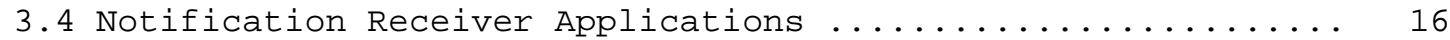

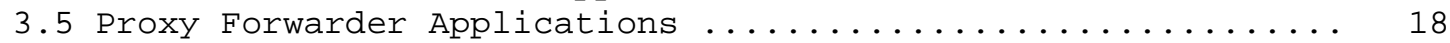

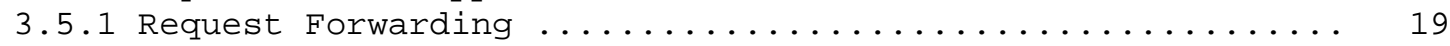

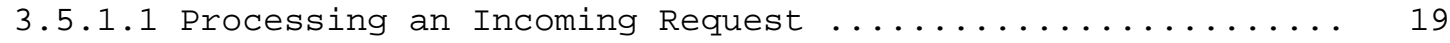

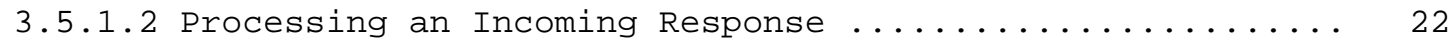

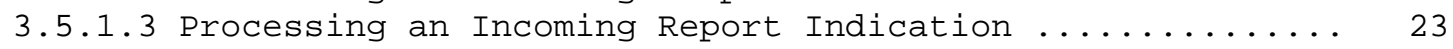

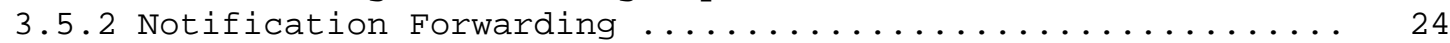

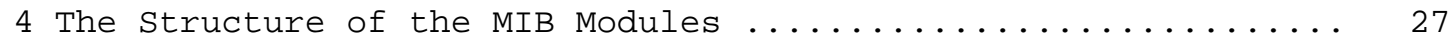

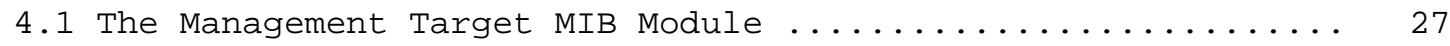

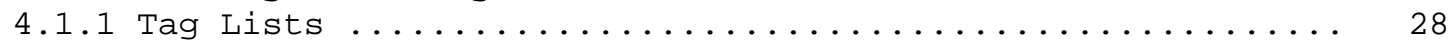

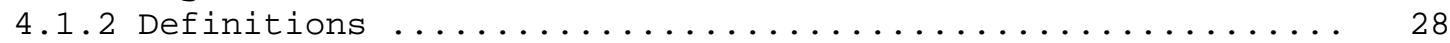

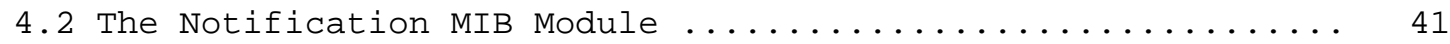

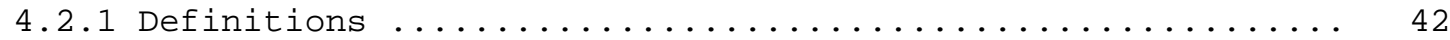

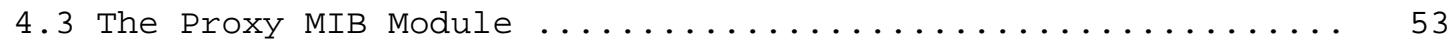

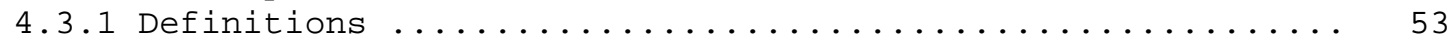

5 Identification of Management Targets in Notification

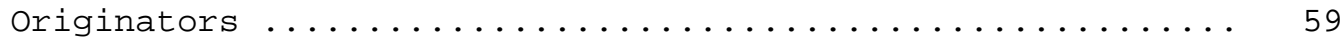

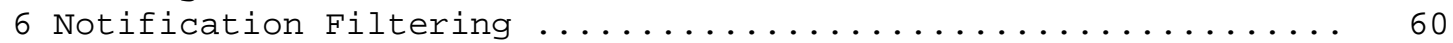

7 Management Target Translation in Proxy Forwarder

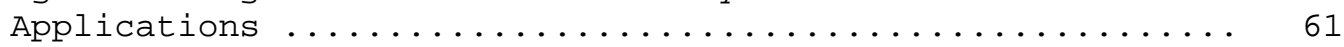

7.1 Management Target Translation for Request Forwarding ...... 61

7.2 Management Target Translation for Notification Forwarding

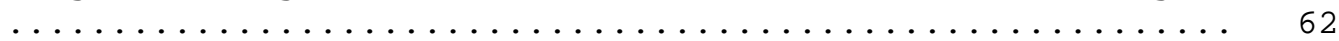

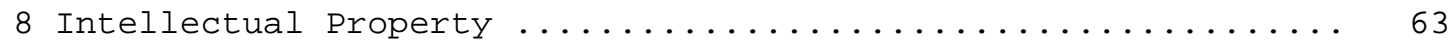

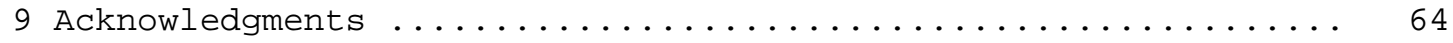

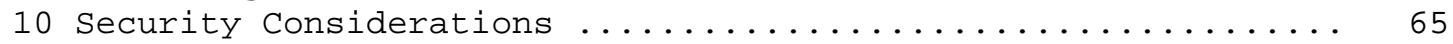

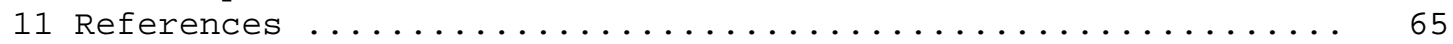

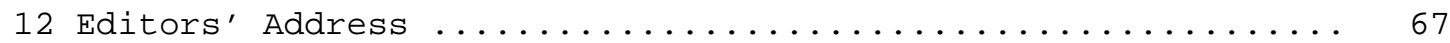

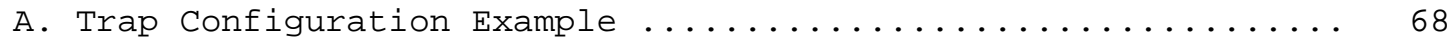

B. Full Copyright Statement ........................ 70

1. Overview

This document describes five types of SNMP applications:

- Applications which initiate SNMP Get, GetNext, GetBulk, and/or set requests, called 'command generators.'

- Applications which respond to SNMP Get, GetNext, GetBulk, and/or set requests, called 'command responders.'

- Applications which generate notifications, called 'notification originators.' 
- Applications which receive notifications, called 'notification receivers.'

- Applications which forward SNMP Get, GetNext, GetBulk, and/or Set requests or notifications, called 'proxy forwarder.'

Note that there are no restrictions on which types of applications may be associated with a particular SNMP engine. For example, a single SNMP engine may, in fact, be associated with both command generator and command responder applications.

\subsection{Command Generator Applications}

A command generator application initiates SNMP Get, GetNext, GetBulk, and/or set requests, as well as processing the response to a request which it generated.

\subsection{Command Responder Applications}

A command responder application receives SNMP Get, GetNext, GetBulk, and/or set requests destined for the local system as indicated by the fact that the contextEngineID in the received request is equal to that of the local engine through which the request was received. The command responder application will perform the appropriate protocol operation, using access control, and will generate a response message to be sent to the request's originator.

\subsection{Notification Originator Applications}

A notification originator application conceptually monitors a system for particular events or conditions, and generates Trap and/or Inform messages based on these events or conditions. A notification originator must have a mechanism for determining where to send messages, and what SNMP version and security parameters to use when sending messages. A mechanism and MIB module for this purpose is provided in this document.

\subsection{Notification Receiver Applications}

A notification receiver application listens for notification messages, and generates response messages when a message containing an Inform PDU is received.

\subsection{Proxy Forwarder Applications}

A proxy forwarder application forwards SNMP messages. Note that implementation of a proxy forwarder application is optional. The sections describing proxy $(4.5,5.3$, and 8$)$ may be skipped for 
implementations that do not include a proxy forwarder application.

The term "proxy" has historically been used very loosely, with multiple different meanings. These different meanings include (among others):

(1) the forwarding of SNMP requests to other SNMP entities without regard for what managed object types are being accessed; for example, in order to forward an SNMP request from one transport domain to another, or to translate SNMP requests of one version into SNMP requests of another version;

(2) the translation of SNMP requests into operations of some non-SNMP management protocol; and

(3) support for aggregated managed objects where the value of one managed object instance depends upon the values of multiple other (remote) items of management information.

Each of these scenarios can be advantageous; for example, support for aggregation of management information can significantly reduce the bandwidth requirements of large-scale management activities. However, using a single term to cover multiple different scenarios causes confusion.

To avoid such confusion, this document uses the term "proxy" with a much more tightly defined meaning. The term "proxy" is used in this document to refer to a proxy forwarder application which forwards either SNMP requests, notifications, and responses without regard for what managed objects are contained within requests or notifications. This definition is most closely related to the first definition above. Note, however, that in the SNMP architecture [RFC2271], a proxy forwarder is actually an application, and need not be associated with what is traditionally thought of as an SNMP agent.

Specifically, the distinction between a traditional SNMP agent and a proxy forwarder application is simple:

- a proxy forwarder application forwards requests and/or notifications to other SNMP engines according to the context, and irrespective of the specific managed object types being accessed, and forwards the response to such previously forwarded messages back to the SNMP engine from which the original message was received;

- in contrast, the command responder application that is part of what is traditionally thought of as an SNMP agent, and which processes SNMP requests according to the (names of the) 
individual managed object types and instances being accessed, is NOT a proxy forwarder application from the perspective of this document.

Thus, when a proxy forwarder application forwards a request or notification for a particular contextEngineID / contextName pair, not only is the information on how to forward the request specifically associated with that context, but the proxy forwarder application has no need of a detailed definition of a MIB view (since the proxy forwarder application forwards the request irrespective of the managed object types).

In contrast, a command responder application must have the detailed definition of the MIB View, and even if it needs to issue requests to other entities, via SNMP or otherwise, that need is dependent on the individual managed object instances being accessed (i.e., not only on the context).

Note that it is a design goal of a proxy forwarder application to act as an intermediary between the endpoints of a transaction. In particular, when forwarding Inform requests, the associated response is forwarded when it is received from the target to which the Inform request was forwarded, rather than generating a response immediately when an Inform request is received.

\section{Management Targets}

Some types of applications (notification generators and proxy forwarders in particular) require a mechanism for determining where and how to send generated messages. This document provides a mechanism and MIB module for this purpose. The set of information that describes where and how to send a message is called a 'Management Target', and consists of two kinds of information:

- Destination information, consisting of a transport domain and a transport address. This is also termed a transport endpoint.

- SNMP parameters, consisting of message processing model, security model, security level, and security name information.

The SNMP-TARGET-MIB module described later in this document contains one table for each of these types of information. There can be a many-to-many relationship in the MIB between these two types of information. That is, there may be multiple transport endpoints associated with a particular set of SNMP parameters, or a particular transport endpoint may be associated with several sets of SNMP parameters. 


\section{Elements of Procedure}

The following sections describe the procedures followed by each type of application when generating messages for transmission or when processing received messages. Applications communicate with the Dispatcher using the abstract service interfaces defined in [RFC2271].

\subsection{Command Generator Applications}

A command generator initiates an SNMP request by calling the Dispatcher using the following abstract service interface:

statusInformation $=$
sendPdu(
IN transportDomain
IN transportAddress
IN messageProcessingModel
IN securityModel
IN securityName
IN securityLevel
IN contextEngineID
IN contextName
IN pduVersion
IN $\quad$ PDU
IN expectResponse

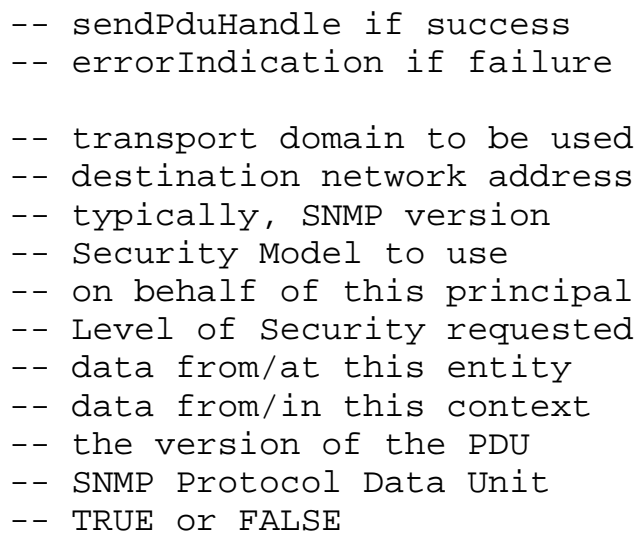

Where:

- The transportDomain is that of the destination of the message.

- The transportAddress is that of the destination of the message.

- The messageProcessingModel indicates which Message Processing Model the application wishes to use.

- The securitymodel is the security model that the application wishes to use.

- The securityName is the security model independent name for the principal on whose behalf the application wishes the message is to be generated.

- The securitylevel is the security level that the application wishes to use. 
- The contextEngineID is provided by the command generator if it wishes to explicitly specify the location of the management information it is requesting.

- The contextName is provided by the command generator if it wishes to explicitly specify the local context name for the management information it is requesting.

- The pduVersion indicates the version of the PDU to be sent.

- The PDU is a value constructed by the command generator containing the management operation that the command generator wishes to perform.

- The expectResponse argument indicates that a response is expected.

The result of the sendPdu interface indicates whether the PDU was successfully sent. If it was successfully sent, the returned value will be a sendPduHandle. The command generator should store the sendPduHandle so that it can correlate a response to the original request.

The Dispatcher is responsible for delivering the response to a particular request to the correct command generator application. The abstract service interface used is:

$\begin{array}{cl}\text { processResponsePdul } \\ \text { IN } & \text { messageProcessingModel } \\ \text { IN } & \text { securityModel } \\ \text { IN } & \text { securityName } \\ \text { IN } & \text { securityLevel } \\ \text { IN } & \text { contextEngineID } \\ \text { IN } & \text { contextName } \\ \text { IN } & \text { pduVersion } \\ \text { IN } & \text { PDU } \\ \text { IN } & \text { statusInformation } \\ \text { IN } & \text { sendPduHandle } \\ & \text { ) }\end{array}$

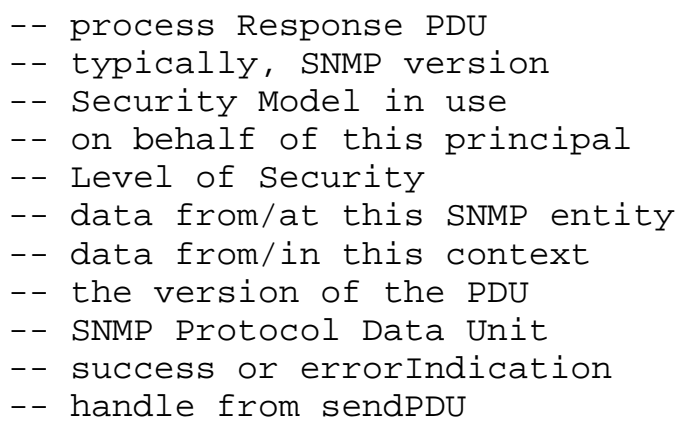

Where:

- The messageProcessingModel is the value from the received response.

- The securitymodel is the value from the received response.

- The securityName is the value from the received response. 
- The securitylevel is the value from the received response.

- The contextEngineID is the value from the received response.

- The contextName is the value from the received response.

- The pduVersion indicates the version of the PDU in the received response.

- The PDU is the value from the received response.

- The statusinformation indicates success or failure in receiving the response.

- The sendPduHandle is the value returned by the sendPdu call which generated the original request to which this is a response.

The procedure when a command generator receives a message is as follows:

(1) If the received values of messageProcessingModel, securityModel, securityName, contextEngineID, contextName, and pduVersion are not all equal to the values used in the original request, the response is discarded.

(2) The operation type, request-id, error-status, error-index, and variable-bindings are extracted from the PDU and saved. If the request-id is not equal to the value used in the original request, the response is discarded.

(3) At this point, it is up to the application to take an appropriate action. The specific action is implementation dependent. If the statusInformation indicates that the request failed, an appropriate action might be to attempt to transmit the request again, or to notify the person operating the application that a failure occurred.

\subsection{Command Responder Applications}

Before a command responder application can process messages, it must first associate itself with an SNMP engine. The abstract service interface used for this purpose is: 


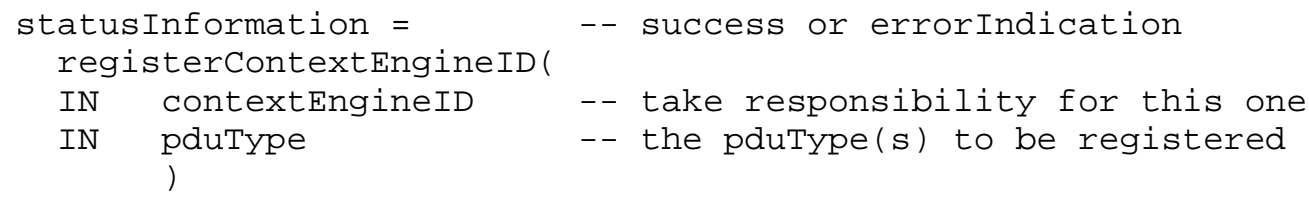

Where:

- The statusinformation indicates success or failure of the registration attempt.

- The contextEngineID is equal to the snmpEngineID of the SNMP engine with which the command responder is registering.

- The pduType indicates a Get, GetNext, GetBulk, or set pdu.

Note that if another command responder application is already registered with an SNMP engine, any further attempts to register with the same contextEngineID and pduType will be denied. This implies that separate command responder applications could register separately for the various pdu types. However, in practice this is undesirable, and only a single command responder application should be registered with an SNMP engine at any given time.

A command responder application can disassociate with an SNMP engine using the following abstract service interface:

unregisterContextEngineID (

IN contextEngineID -- give up responsibility for this one

IN pduType -- the pduType(s) to be unregistered )

Where:

- The contextEngineID is equal to the snmpEngineID of the SNMP engine with which the command responder is cancelling the registration.

- The pduType indicates a Get, GetNext, GetBulk, or set pdu.

Once the command responder has registered with the SNMP engine, it waits to receive SNMP messages. The abstract service interface used for receiving messages is:

$\begin{array}{cl}\text { processPdul } & -- \text { process Request/Notification PDU } \\ \text { IN messageProcessingModel } & -- \text { typically, SNMP version } \\ \text { IN securityModel } & -- \text { Security Model in use } \\ \text { IN securityName } & -- \text { on behalf of this principal }\end{array}$




$\begin{array}{lll}\text { IN } & \text { securityLevel } & -- \text { Level of Security } \\ \text { IN } & \text { contextEngineID } & -- \text { data from/at this SNMP entity } \\ \text { IN } & \text { contextName } & -- \text { data from/in this context } \\ \text { IN } & \text { pduVersion } & -- \text { the version of the PDU } \\ \text { IN } & \text { PDU } & -- \text { SNMP Protocol Data Unit } \\ \text { IN } & \text { maxSizeResponseScopedPDU } & -- \text { maximum size of the Response PDU } \\ \text { IN } & \text { stateReference } & -- \text { reference to state information } \\ & \text { ) } & -- \text { needed when sending a response }\end{array}$

Where:

- The messageProcessingModel indicates which Message Processing Model received and processed the message.

- The securityModel is the value from the received message.

- The securityName is the value from the received message.

- The securitylevel is the value from the received message.

- The contextEngineID is the value from the received message.

- The contextName is the value from the received message.

- The pduVersion indicates the version of the PDU in the received message.

- The PDU is the value from the received message.

- The maxSizeResponseScopedPDU is the maximum allowable size of a ScopedPDU containing a Response PDU (based on the maximum message size that the originator of the message can accept).

- The stateReference is a value which references cached information about each received request message. This value must be returned to the Dispatcher in order to generate a response.

The procedure when a message is received is as follows.

(1) The operation type is determined from the ASN.1 tag value associated with the PDU parameter. The operation type should always be one of the types previously registered by the application.

(2) The request-id is extracted from the PDU and saved. 
(3) If the SNMPV2 operation type is GetBulk, the non-repeaters and max-repetitions values are extracted from the PDU and saved.

(4) The variable-bindings are extracted from the PDU and saved.

(5) The management operation represented by the SNMPv2 operation type is performed with respect to the relevant MIB view within the context named by the contextName, according to the procedures set forth in [RFC1905]. The relevant MIB view is determined by the securitylevel, securityModel, contextName, securityName, and SNMPv2 operation type. To determine whether a particular object instance is within the relevant MIB view, the following abstract service interface is called:

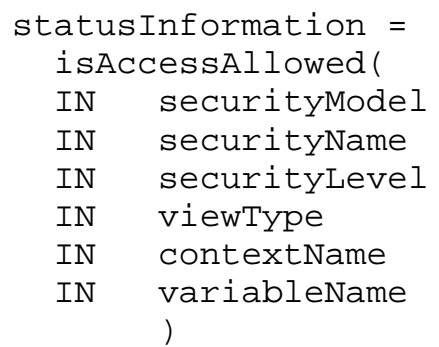

Where:

- The securitymodel is the value from the received message.

- The securityName is the value from the received message.

- The securitylevel is the value from the received message.

- The viewType indicates whether the PDU type is a read or write operation.

- The contextName is the value from the received message.

- The variableName is the object instance of the variable for which access rights are to be checked.

Normally, the result of the management operation will be a new PDU value, and processing will continue in step (6) below. However, at any time during the processing of the management operation:

- If the isAccessAllowed ASI returns a noSuchView, noAccessentry, or noGroupName error, processing of the management operation is halted, a PDU value is contructed using the values from the originally received PDU, but 
replacing the error_status with an authorizationError code, and error_index value of 0 , and control is passed to step (6) below.

- If the isAccessAllowed ASI returns an otherError, processing of the management operation is halted, a different PDU value is contructed using the values from the originally received PDU, but replacing the error_status with a genError code, and control is passed to step (6) below.

- If the isAccessAllowed ASI returns a noSuchcontext error, processing of the management operation is halted, no result PDU is generated, the snmpUnknownContexts counter is incremented, and control is passed to step (6) below.

- If the context named by the contextName parameter is unavailable, processing of the management operation is halted, no result $\mathrm{PDU}$ is generated, the snmpUnavailableContexts counter is incremented, and control is passed to step (6) below.

(6) The Dispatcher is called to generate a response or report message. The abstract service interface is:

$\begin{aligned} & \text { returnesponsePdu( } \\ & \text { IN } \text { messageProcessingModel } \\ & \text { IN } \text { securityModel } \\ & \text { IN } \text { securityName } \\ & \text { IN } \text { securityLevel } \\ & \text { IN } \text { contextEngineID } \\ & \text { IN } \text { contextName } \\ & \text { IN } \text { pduVersion } \\ & \text { IN } \text { PDU } \\ & \text { IN } \text { maxSizeResponseScopedPDU } \\ & \text { IN } \text { stateReference } \\ & \\ & \text { IN } \text { statusinformation } \\ & \text { ) }\end{aligned}$

Where:

- The messageProcessingModel is the value from the processPdu call.

- The securitymodel is the value from the processPdu call.

- The securityName is the value from the processPdu call.

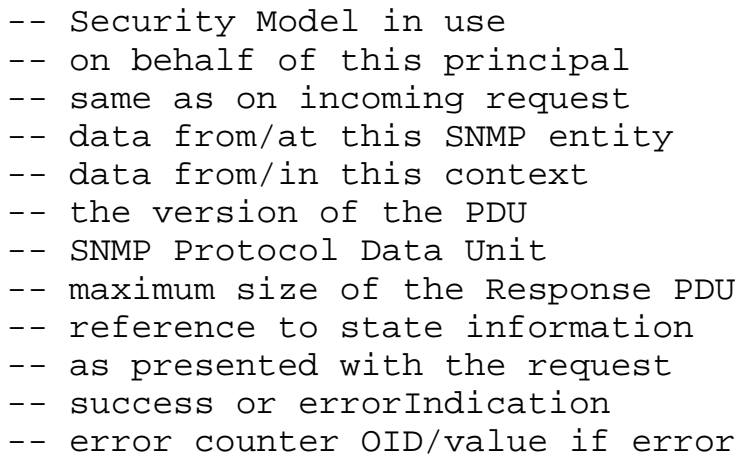


- The securitylevel is the value from the processpdu call.

- The contextEngineID is the value from the processPdu call.

- The contextName is the value from the processPdu call.

- The pduVersion indicates the version of the PDU to be returned. If no result PDU was generated, the pduVersion is an undefined value.

- The PDU is the result generated in step (5) above. If no result $\mathrm{PDU}$ was generated, the PDU is an undefined value.

- The maxSizeResponseScopedPDU is a local value indicating the maximum size of a ScopedPDU that the application can accept.

- The stateReference is the value from the processPdu call.

- The statusinformation either contains an indication that no error occurred and that a response should be generated, or contains an indication that an error occurred along with the OID and counter value of the appropriate error counter object.

Note that a command responder application should always call the returnResponsePdu abstract service interface, even in the event of an error such as a resource allocation error. In the event of such an error, the PDU value passed to returnResponsePdu should contain appropriate values for errorstatus and errorIndex.

\subsection{Notification Originator Applications}

A notification originator application generates SNMP notification messages. A notification message may, for example, contain an SNMPv2-Trap PDU or an Inform PDU. However, a particular implementation is not required to be capable of generating both types of messages.

Notification originator applications require a mechanism for identifying the management targets to which notifications should be sent. The particular mechanism used is implementation dependent. However, if an implementation makes the configuration of management targets SNMP manageable, it MUST use the SNMP-TARGET-MIB module described in this document.

When a notification originator wishes to generate a notification, it must first determine in which context the information to be conveyed in the notification exists, i.e., it must determine the contextEngineID and contextName. It must then determine the set of 
management targets to which the notification should be sent. The application must also determine, for each management target, whether the notification message should contain an SNMPv2-Trap PDU or Inform PDU, and if it is to contain an Inform PDU, the number of retries and retransmission algorithm.

The mechanism by which a notification originator determines this information is implementation dependent. Once the application has determined this information, the following procedure is performed for each management target:

(1) Any appropriate filtering mechanisms are applied to determine whether the notification should be sent to the management target. If such filtering mechanisms determine that the notification should not be sent, processing continues with the next management target. Otherwise,

(2) The appropriate set of variable-bindings is retrieved from local MIB instrumentation within the relevant MIB view. The relevant MIB view is determined by the securitylevel, securityModel, contextName, and securityName of the management target. To determine whether a particular object instance is within the relevant MIB view, the isAccessAllowed abstract service interface is used, in the same manner as described in the preceding section. If the statusInformation returned by isAccessAllowed does not indicate accessAllowed, the notification is not sent to the management target.

(3) A PDU is constructed using a locally unique request-id value, an operation type of SNMPv2-Trap or Inform, an error-status and error-index value of 0 , and the variable-bindings supplied previously in step (2).

(4) If the notification contains an SNMPv2-Trap PDU, the Dispatcher is called using the following abstract service interface:

statusinformation $=$
sendPdu(
IN transportDomain
IN transportAddress
IN messageProcessingModel
IN securityModel
IN securityName
IN securityLevel
IN contextEngineID
IN contextName
IN pduVersion

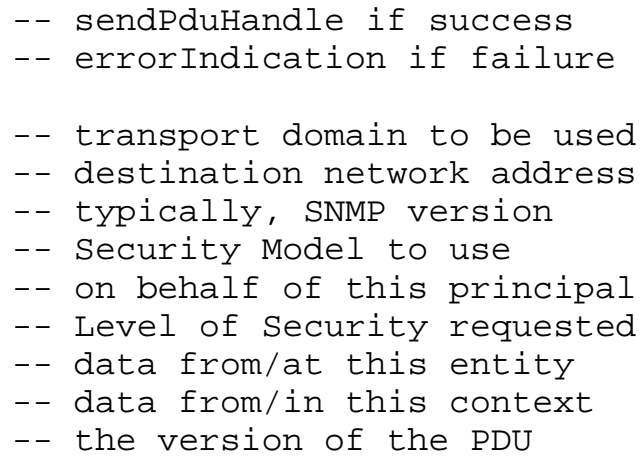




$\begin{array}{lll}\text { IN PDU } & -- \text { SNMP Protocol Data Unit } \\ \text { IN expectResponse } & -- \text { TRUE or FALSE }\end{array}$

Where:

- The transportDomain is that of the management target.

- The transportAddress is that of the management target.

- The messageProcessingModel is that of the management target.

- The securityModel is that of the management target.

- The securityName is that of the management target.

- The securityLevel is that of the management target.

- The contextEngineID is the value originally determined for the notification.

- The contextName is the value originally determined for the notification.

- The pduVersion is the version of the PDU to be sent.

- The PDU is the value constructed in step (3) above.

- The expectResponse argument indicates that no response is expected.

Otherwise,

(5) If the notification contains an Inform PDU, then:

a) The Dispatcher is called using the sendPdu abstract service interface as described in step (4) above, except that the expectResponse argument indicates that a response is expected.

b) The application caches information about the management target.

c) If a response is received within an appropriate time interval from the transport endpoint of the management target, the notification is considered acknowledged and the cached information is deleted. Otherwise, 
d) If a response is not received within an appropriate time period, or if a report indication is received, information about the management target is retrieved from the cache, and steps a) through d) are repeated. The number of times these steps are repeated is equal to the previously determined retry count. If this retry count is exceeded, the acknowledgement of the notification is considered to have failed, and processing of the notification for this management target is halted.

Responses to Inform PDU notifications will be received via the processResponsePDU abstract service interface.

3.4. Notification Receiver Applications

Notification receiver applications receive SNMP Notification messages from the Dispatcher. Before any messages can be received, the notification receiver must register with the Dispatcher using the registercontextEngineID abstract service interface. The parameters used are:

- The contextEngineID is an undefined 'wildcard' value. Notifications are delivered to a registered notification receiver regardless of the contextEngineID contained in the notification message.

- The pduType indicates the type of notifications that the application wishes to receive (for example, SNMPv2-Trap PDUs or Inform PDUs).

Once the notification receiver has registered with the Dispatcher, messages are received using the processPdu abstract service interface. Parameters are:

- The messageProcessingModel indicates which Message Processing Model received and processed the message.

- The securityModel is the value from the received message.

- The securityName is the value from the received message.

- The securityLevel is the value from the received message.

- The contextEngineID is the value from the received message.

- The contextName is the value from the received message. 
- The pduVersion indicates the version of the PDU in the received message.

- The PDU is the value from the received message.

- The maxSizeResponseScopedPDU is the maximum allowable size of a ScopedPDU containing a Response PDU (based on the maximum message size that the originator of the message can accept).

- If the message contains an SNMPV2-Trap PDU, the stateReference is undefined and unused. Otherwise, the stateReference is a value which references cached information about the notification. This value must be returned to the Dispatcher in order to generate a response.

When an SNMPV2-Trap PDU is delivered to a notification receiver application, it first extracts the SNMP operation type, request-id, error-status, error-index, and variable-bindings from the PDU. After this, processing depends on the particular implementation.

When an Inform PDU is received, the notification receiver application follows the following procedure:

(1) The SNMPv2 operation type, request-id, error-status, error-index, and variable-bindings are extracted from the PDU.

(2) A Response PDU is constructed using the extracted request-id and variable-bindings, and with error-status and error-index both set to 0 .

(3) The Dispatcher is called to generate a response message using the returnResponsePdu abstract service interface. Parameters are:

- The messageProcessingModel is the value from the processPdu call.

- The securityModel is the value from the processPdu call.

- The securityName is the value from the processPdu call.

- The securitylevel is the value from the processPdu call.

- The contextEngineID is the value from the processPdu call.

- The contextName is the value from the processPdu call.

- The pduVersion indicates the version of the PDU to be returned. 
- The PDU is the result generated in step (2) above.

- The maxSizeresponseScopedPDU is a local value indicating the maximum size of a ScopedPDU that the application can accept.

- The stateReference is the value from the processPdu call.

- The statusInformation indicates that no error occurred and that a response should be generated.

\subsection{Proxy Forwarder Applications}

A proxy forwarder application deals with forwarding SNMP messages. There are four basic types of messages which a proxy forwarder application may need to forward. These are grouped according to the PDU type contained in a message, or according to whether a report indication is contained in the message. The four basic types of messages are:

- Those containing PDU types which were generated by a command generator application (for example, Get, GetNext, GetBulk, and Set PDU types). These deal with requesting or modifying information located within a particular context.

- Those containing PDU types which were generated by a notification originator application (for example, SNMPv2-Trap and Inform PDU types). These deal with notifications concerning information located within a particular context.

- Those containing a Response PDU type. Forwarding of Response PDUs always occurs as a result of receiving a response to a previously forwarded message.

- Those containing a report indication. Forwarding of report indications always occurs as a result of receiving a report indication for a previously forwarded message.

For the first type, the proxy forwarder's role is to deliver a request for management information to an SNMP engine which is "closer" or "downstream in the path" to the SNMP engine which has access to that information, and to deliver the response containing the information back to the SNMP engine from which the request was received. The context information in a request is used to determine which SNMP engine has access to the requested information, and this is used to determine where and how to forward the request. 
For the second type, the proxy forwarder's role is to determine which SNMP engines should receive notifications about management

information from a particular location. The context information in a notification message determines the location to which the information contained in the notification applies. This is used to determine which SNMP engines should receive notification about this information.

For the third type, the proxy forwarder's role is to determine which previously forwarded request or notification (if any) the response matches, and to forward the response back to the initiator of the request or notification.

For the fourth type, the proxy forwarder's role is to determine which previously forwarded request or notification (if any) the report indication matches, and to forward the report indication back to the initiator of the request or notification.

When forwarding messages, a proxy forwarder application must perform a translation of incoming management target information into outgoing management target information. How this translation is performed is implementation specific. In many cases, this will be driven by a preconfigured translation table. If a proxy forwarder application makes the contents of this table SNMP manageable, it MUST use the SNMP-PROXY-MIB module defined in this document.

\subsubsection{Request Forwarding}

There are two phases for request forwarding. First, the incoming request needs to be passed through the proxy application. Then, the resulting response needs to be passed back. These phases are described in the following two sections.

\subsubsection{Processing an Incoming Request}

A proxy forwarder application that wishes to forward request messages must first register with the Dispatcher using the registerContextEngineID abstract service interface. The proxy forwarder must register each contextEngineID for which it wishes to forward messages, as well as for each pduType. Note that as the configuration of a proxy forwarder is changed, the particular contextEngineID values for which it is forwarding may change. The proxy forwarder should call the registercontextEngineID and unregisterContextEngineID abstract service interfaces as needed to reflect its current configuration. 
A proxy forwarder application should never attempt to register a value of contextEngineID which is equal to the snmpEngineID of the SNMP engine to which the proxy forwarder is associated.

Once the proxy forwarder has registered for the appropriate contextEngineId values, it can start processing messages. The following procedure is used:

(1) A message is received using the processPdu abstract service interface. The incoming management target information received from the processPdu interface is translated into outgoing management target information. Note that this translation may vary for different values of contextEngineID and/or contextName. The translation should result in a single management target.

(2) If appropriate outgoing management target information cannot be found, the proxy forwarder increments the snmpProxyDrops counter [RFC1907], and then calls the Dispatcher using the returnResponsePdu abstract service interface. Parameters are:

- The messageProcessingModel is the value from the processPdu call.

- The securitymodel is the value from the processPdu call.

- The securityName is the value from the processPdu call.

- The securitylevel is the value from the processPdu call.

- The contextEngineID is the value from the processPdu call.

- The contextName is the value from the processPdu call.

- The pduVersion is the value from the processPdu call.

- The PDU is an undefined value.

- The maxSizeResponseScopedPDU is a local value indicating the maximum size of a ScopedPDU that the application can accept.

- The stateReference is the value from the processpdu call.

- The statusinformation indicates that an error occurred and includes the OID and value of the snmpProxyDrops object.

Processing of the message stops at this point. Otherwise, 
(3) A new PDU is constructed. A unique value of request-id should be used in the new PDU (this value will enable a subsequent response message to be correlated with this request). The remainder of the new PDU is identical to the received PDU, unless the incoming SNMP version is SNMPV2 or SNMPv3 and the outgoing SNMP version is SNMPv1, in which case the proxy forwarder must apply the translation rules as documented in [RFC1908].

(4) The proxy forwarder calls the Dispatcher to generate the forwarded message, using the sendPdu abstract service interface. The parameters are:

- The transportDomain is that of the outgoing management target.

- The transportAddress is that of the outgoing management target.

- The messageProcessingModel is that of the outgoing management target.

- The securitymodel is that of the outgoing management target.

- The securityName is that of the outgoing management target.

- The securitylevel is that of the outgoing management target.

- The contextEngineID is the value originally received.

- The contextName is the value originally received.

- The pduVersion is the version of the PDU to be sent.

- The PDU is the value constructed in step (3) above.

- The expectResponse argument indicates that a response is expected. If the sendPdu call is unsuccessful, the proxy forwarder performs the steps described in (2) above. Otherwise:

(5) The proxy forwarder caches the following information in order to match an incoming response to the forwarded request:

- The sendPduHandle returned from the call to sendPdu,

- The request-id from the received PDU.

- the contextEngineid, 
- the contextName,

- the stateReference,

- the incoming management target information,

- the outgoing management information,

- any other information needed to match an incoming response to the forwarded request.

If this information cannot be cached (possibly due to a lack of resources), the proxy forwarder performs the steps described in (2) above. Otherwise:

(6) Processing of the request stops until a response to the forwarded request is received, or until an appropriate time interval has expired. If this time interval expires before a response has been received, the cached information about this request is removed.

3.5.1.2. Processing an Incoming Response

A proxy forwarder follows the following procedure when an incoming response is received:

(1) The incoming response is received using the processResponsePdu interface. The proxy forwarder uses the received parameters to locate an entry in its cache of pending forwarded requests. This is done by matching the received parameters with the cached values of sendPduHandle, contextEngineID, contextName, outgoing management target information, and the request-id contained in the received PDU (the proxy forwarder must extract the request-id for this purpose). If an appropriate cache entry cannot be found, processing of the response is halted. Otherwise:

(2) The cache information is extracted, and removed from the cache.

(3) A new Response PDU is constructed, using the request-id value from the original forwarded request (as extracted from the cache). All other values are identical to those in the received Response PDU.

(4) If the incoming SNMP version is SNMPv1 and the outgoing SNMP version is SNMPv2 or SNMPv3, the proxy forwarder must apply the translation rules documented in [RFC1908].

(5) The proxy forwarder calls the Dispatcher using the returnResponsePdu abstract service interface. Parameters are: 
- The messageProcessingModel indicates the Message Processing Model by which the original incoming message was processed.

- The securitymodel is that of the original incoming management target extracted from the cache.

- The securityName is that of the original incoming management target extracted from the cache.

- The securitylevel is that of the original incoming management target extracted from the cache.

- The contextEngineID is the value extracted from the cache.

- The contextName is the value extracted from the cache.

- The pduVersion indicates the version of the PDU to be returned.

- The PDU is the (possibly translated) Response PDU.

- The maxSizeResponseScopedPDU is a local value indicating the maximum size of a ScopedPDU that the application can accept.

- The stateReference is the value extracted from the cache.

- The statusInformation indicates that no error occurred and that a Response PDU message should be generated.

\subsubsection{Processing an Incoming Report Indication}

A proxy forwarder follows the following procedure when an incoming report indication is received:

(1) The incoming report indication is received using the processResponsePdu interface. The proxy forwarder uses the received parameters to locate an entry in its cache of pending forwarded requests. This is done by matching the received parameters with the cached values of sendPduHandle. If an appropriate cache entry cannot be found, processing of the report indication is halted. Otherwise:

(2) The cache information is extracted, and removed from the cache.

(3) If the original incoming management target information indicates SNMPV1, processing of the report indication is halted. 
(4) The proxy forwarder calls the Dispatcher using the returnResponsePdu abstract service interface. Parameters are:

- The messageProcessingModel indicates the Message Processing Model by which the original incoming message was processed.

- The securitymodel is that of the original incoming management target extracted from the cache.

- The securityName is that of the original incoming management target extracted from the cache.

- The securitylevel is that of the original incoming management target extracted from the cache.

- The contextEngineID is the value extracted from the cache.

- The contextName is the value extracted from the cache.

- The pduVersion indicates the version of the PDU to be returned.

- The PDU is unused.

- The maxSizeresponseScopedPDU is a local value indicating the maximum size of a ScopedPDU that the application can accept.

- The stateReference is the value extracted from the cache.

- The statusInformation contain the contextEngineID, contextName, counter OID, and counter value received in the report indication.

\subsubsection{Notification Forwarding}

A proxy forwarder receives notifications in the same manner as a notification receiver application, using the processpdu abstract service interface. The following procedure is used when a notification is received:

(1) The incoming management target information received from the processPdu interface is translated into outgoing management target information. Note that this translation may vary for different values of contextEngineId and/or contextName. The translation may result in multiple management targets. 
(2) If appropriate outgoing management target information cannot be found and the notification was a Trap, processing of the notification is halted. If appropriate outgoing management target information cannot be found and the notification was an Inform, the proxy forwarder increments the snmpProxyDrops object, and calls the Dispatcher using the returnResponsePdu abstract service interface.

The parameters are:

- The messageProcessingModel is the received value.

- The securityModel is the received value.

- The securityName is the received value.

- The securitylevel is the received value.

- The contextEngineID is the received value.

- The contextName is the received value.

- The pduVersion is the received value.

- The PDU is an undefined and unused value.

- The maxSizeResponseScopedPDU is a local value indicating the maximum size of a ScopedPDU that the application can accept.

- The stateReference is the received value.

- The statusInformation indicates that an error occurred and that a Report message should be generated.

Processing of the message stops at this point. Otherwise,

(3) The proxy forwarder generates a notification using the procedures described in the preceding section on Notification Originators, with the following exceptions:

- The contextEngineID and contextName values from the original received notification are used.

- The outgoing management targets previously determined are used.

- No filtering mechanisms are applied. 
- The variable-bindings from the original received notification are used, rather than retrieving variable-bindings from local MIB instrumentation. In particular, no access-control is applied to these variable-bindings.

- If for any of the outgoing management targets, the incoming SNMP version is SNMPV1 and the outgoing SNMP version is SNMPV2 or SNMPv3, the proxy forwarder must apply the translation rules as documented in [RFC1908].

- If for any of the outgoing management targets, the incoming SNMP version is SNMPV2 or SNMPV3, and the outgoing SNMP version is SNMPV1, this outgoing management target is not used when generating the forwarded notifications.

(4) If the original received notification contains an SNMPv2-Trap PDU, processing of the notification is now completed. Otherwise, the original received notification must contain an Inform PDU, and processing continues.

(5) If the forwarded notifications included any Inform PDUs, processing continues when the procedures described in the section for Notification Originators determine that either:

- None of the generated notifications containing Inform PDUs have been successfully acknowledged within the longest of the time intervals, in which case processing of the original notification is halted, or,

- At least one of the generated notifications containing Inform PDUs is successfully acknowledged, in which case a response to the original received notification containing an Inform PDU is generated as described in the following steps.

(6) A Response PDU is constructed, using the values of request-id and variable-bindings from the original received Inform PDU, and error-status and error-index values of 0 .

(7) The Dispatcher is called using the returnResponsePdu abstract service interface. Parameters are:

- The messageProcessingModel is the originally received value.

- The securityModel is the originally received value.

- The securityName is the originally received value.

- The securitylevel is the originally received value. 
- The contextEngineID is the originally received value.

- The contextName is the originally received value.

- The pduVersion indicates the version of the PDU constructed in step (6) above.

- The PDU is the value constructed in step (6) above.

- The maxSizeResponseScopedPDU is a local value indicating the maximum size of a ScopedPDU that the application can accept.

- The stateReference is the originally received value.

- The statusinformation indicates that no error occurred and that a Response PDU message should be generated.

4. The structure of the MIB Modules

There are three separate MIB modules described in this document, the management target MIB, the notification MIB, and the proxy MIB. The following sections describe the structure of these three MIB modules.

The use of these MIBs by particular types of applications is described later in this document:

- The use of the management target MIB and the notification MIB in notification originator applications is described in section 6 .

- The use of the notification MIB for filtering notifications in notification originator applications is described in section 7 .

- The use of the management target MIB and the proxy MIB in proxy forwarding applications is described in section 8 .

\subsection{The Management Target MIB Module}

The SNMP-TARGET-MIB module contains objects for defining management targets. It consists of two tables and conformance/compliance statements.

The first table, the snmpTargetAddrTable, contains information about transport domains and addresses. It also contains an object, snmpTargetAddrTagList, which provides a mechanism for grouping entries. 
The second table, the snmpTargetParamstable, contains information about SNMP version and security information to be used when sending messages to particular transport domains and addresses.

\subsubsection{Tag Lists}

The snmpTargetAddrTagList object is used for grouping entries in the snmpTargetAddrTable. The value of this object contains a list of tag values which are used to select target addresses to be used for a particular operation.

A tag value, which may also be used in MIB objects other than snmpTargetAddrTagList, is an arbitrary string of octets, but may not contain a delimiter character. Delimiter characters are defined to be one of the following characters:

- An ASCII space character $(0 \times 20)$.

- An ASCII TAB character (0x09).

- An ASCII carriage return (CR) character (0xOD).

- An ASCII line feed (LF) character (0x0B).

In addition, a tag value may not have a zero length. Generally, a particular MIB object may contain either

- a single tag value, in which case the value of the MIB object may not contain a delimiter character, or:

- a MIB object may contain a list of tag values, separated by single delimiter characters.

For a list of tag values, these constraints imply certain restrictions on the value of a MIB object:

- There cannot be a leading or trailing delimiter character.

- There cannot be multiple adjacent delimiter charaters.

\subsubsection{Definitions}

SNMP-TARGET-MIB DEFINITIONS : := BEGIN

IMPORTS

TEXTUAL-CONVENTION,

MODULE-IDENTITY,

OBJECT-TYPE, 
snmpModules,
Integer 32

FROM SNMPV2-SMI

TDomain,

TAddress,

TimeInterval,

Rowstatus,

StorageType,

TestAndIncr

FROM SNMPV2-TC

SnmpsecurityModel,

SnmpMessageProcessingModel,

SnmpsecurityLevel,

SnmpAdminstring

FROM SNMP-FRAMEWORK-MIB

OBJECT-GROUP

FROM SNMPV2-CONE;

snmpTargetMIB MODULE-IDENTITY

LAST-UPDATED "9711210000Z"

ORGANIZATION "IETF SNMPv3 Working Group"

CONTACT-INFO

"WG-email: snmpv3etis.com

Subscribe: majordomodtis.com

In message body: subscribe snmpv3

Chair: Russ Mundy

Trusted Information systems

Postal: $\quad 3060$ Washington Rd

Glenwood MD 21738

USA

Email: mundy@tis.com

Phone: $\quad+1-301-854-6889$

Co-editor: David B. Levi

SNMP Research, Inc.

Postal: $\quad 3001$ Kimberlin Heights Road

Knoxville, TN 37920-9716

E-mail: leviesnmp.com

Phone: +1 4235731434

Co-editor: Paul Meyer

Secure Computing Corporation

Postal: 2675 Long Lake Road

Roseville, MN 55113

E-mail: paul_meyerdsecurecomputing.com

Levi, et. al.

Standards Track

[Page 29] 


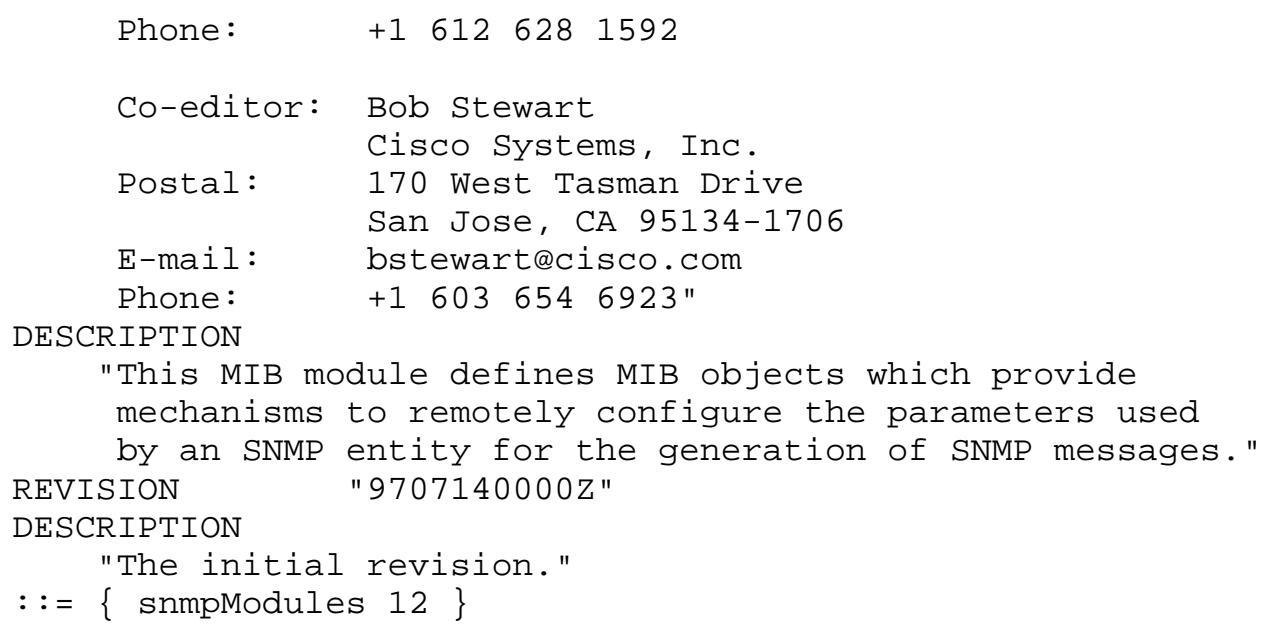


Note that when this TC is used for an object that is used or envisioned to be used as an index, then a SIZE restriction must be specified so that the number sub-identifiers for any object instance do not exceed the limit of 128, as defined by [RFC1905].

An object of this type contains a single tag value which is used to select a set of entries in a table. A tag value is an arbitrary string of octets, but may not contain a delimiter character. Delimiter characters are defined to be one of the following:

- An ASCII space character (0x20).

- An ASCII TAB character (0x09).

- An ASCII carriage return (CR) character (0x0D).

- An ASCII line feed (LF) character (0x0B).

Delimiter characters are used to separate tag values in a tag list. An object of this type may only contain a single tag value, and so delimiter characters are not allowed in a value of this type.

Some examples of valid tag values are:

$-'$ acme'

- 'router'

- 'host'

The use of a tag value to select table entries is application and MIB specific."

SYNTAX OCTET STRING (SIZE (0..255))

SnmpTagList : := TEXTUAL-CONVENTION

DISPLAY-HINT "255a"

STATUS current

DESCRIPTION

"An octet string containing a list of tag values. Tag values are preferably in human-readable form.

To facilitate internationalization, this information is represented using the ISO/IEC IS 10646-1 character set, encoded as an octet string using the UTF-8 character encoding scheme described in RFC 2044. 


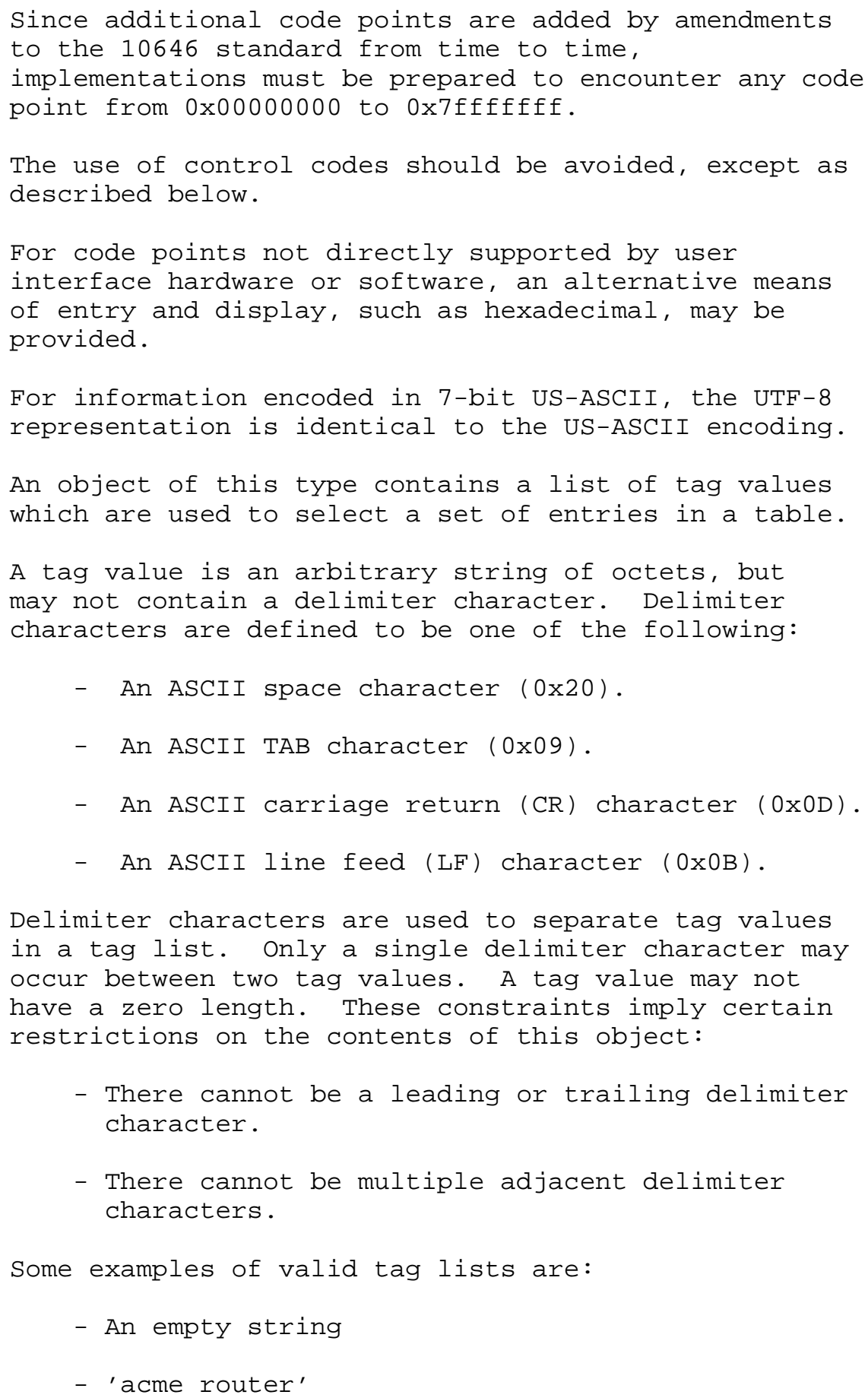


- 'host managerstation'

Note that although a tag value may not have a length of zero, an empty string is still valid. This indicates an empty list (i.e. there are no tag values in the list).

The use of the tag list to select table entries is application and MIB specific. Typically, an application will provide one or more tag values, and any entry which contains some combination of these tag values will be selected." SYNTAX OCTET STRING (SIZE (0..255))

\section{$--$}

-- The snmptargetobjects group

$--$

$--$

snmpTargetSpinLock OBJECT-TYPE

SYNTAX TestAndIncr

MAX-ACCESS read-write

STATUS current

DESCRIPTION

"This object is used to facilitate modification of table entries in the SNMP-TARGET-MIB module by multiple managers. In particular, it is useful when modifying the value of the snmpTargetAddrTagList object.

The procedure for modifying the snmpTargetAddrTaglist object is as follows:

1. Retrieve the value of snmpTargetSpinLock and of snmpTargetAddrTagList.

2. Generate a new value for snmpTargetAddrTagList.

3. Set the value of snmpTargetspinLock to the retrieved value, and the value of snmpTargetAddrTagList to the new value. If the set fails for the snmpTargetSpinLock object, go back to step 1."

$::=\{$ snmptargetobjects 1$\}$

snmpTargetAddrTable OBJECT-TYPE

SYNTAX SEQUENCE OF SnmpTargetAddrEntry

MAX-ACCESS not-accessible

STATUS current 


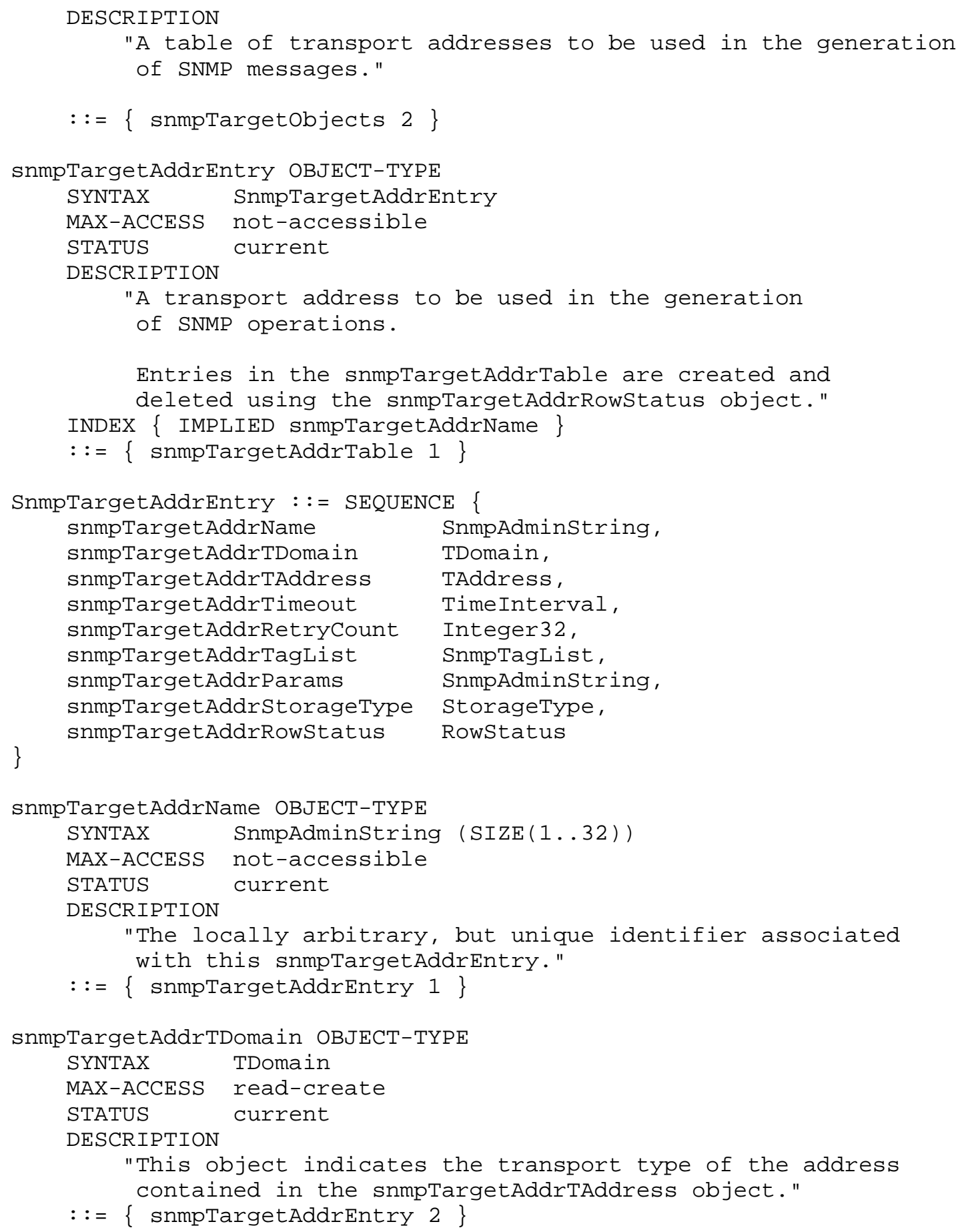




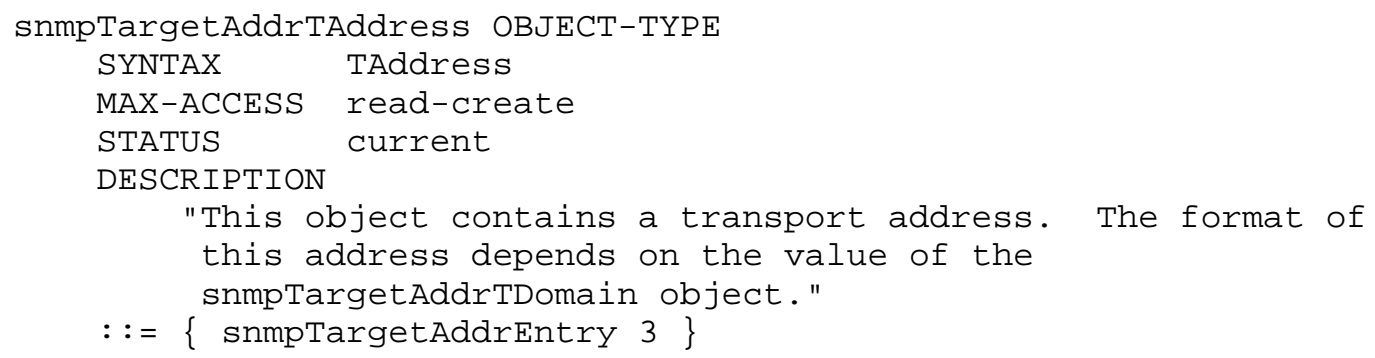


$::=\{$ snmpTargetAddrEntry 5$\}$

snmpTargetAddrTagList OBJECT-TYPE

SYNTAX SnmpTaglist

MAX-ACCESS read-create

STATUS current

DESCRIPTION

"This object contains a list of tag values which are used to select target addresses for a particular operation."

$::=\{$ snmpTargetAddrEntry 6$\}$

snmpTargetAddrParams OBJECT-TYPE

SYNTAX SnmpAdminString (SIZE(1..32))

MAX-ACCESS read-create

STATUS current

DESCRIPTION

"The value of this object identifies an entry in the snmpTargetParamsTable. The identified entry contains SNMP parameters to be used when generating messages to be sent to this transport address."

$::=\{$ snmpTargetAddrEntry 7$\}$

snmpTargetAddrStorageType OBJECT-TYPE

SYNTAX StorageType

MAX-ACCESS read-create

STATUS current

DESCRIPTION

"The storage type for this conceptual row."

$::=\{$ snmpTargetAddrEntry 8$\}$

snmpTargetAddrRowStatus OBJECT-TYPE

SYNTAX Rowstatus

MAX-ACCESS read-create

STATUS current

DESCRIPTION

"The status of this conceptual row.

To create a row in this table, a manager must

set this object to either createAndGo(4) or

createAndWait (5).

Until instances of all corresponding columns are

appropriately configured, the value of the

corresponding instance of the snmpTargetAddrRowStatus

column is 'notReady'.

In particular, a newly created row cannot be made 


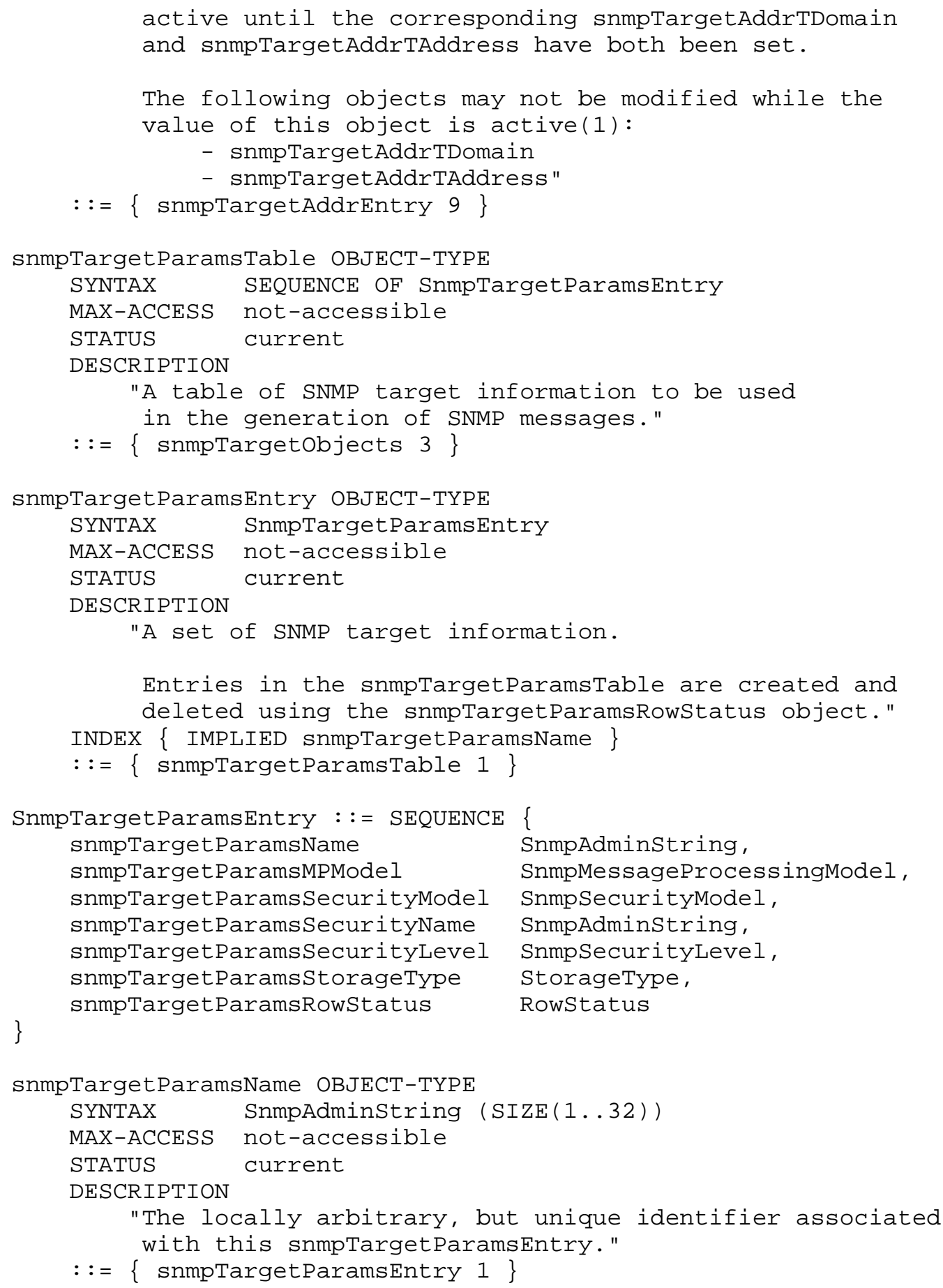




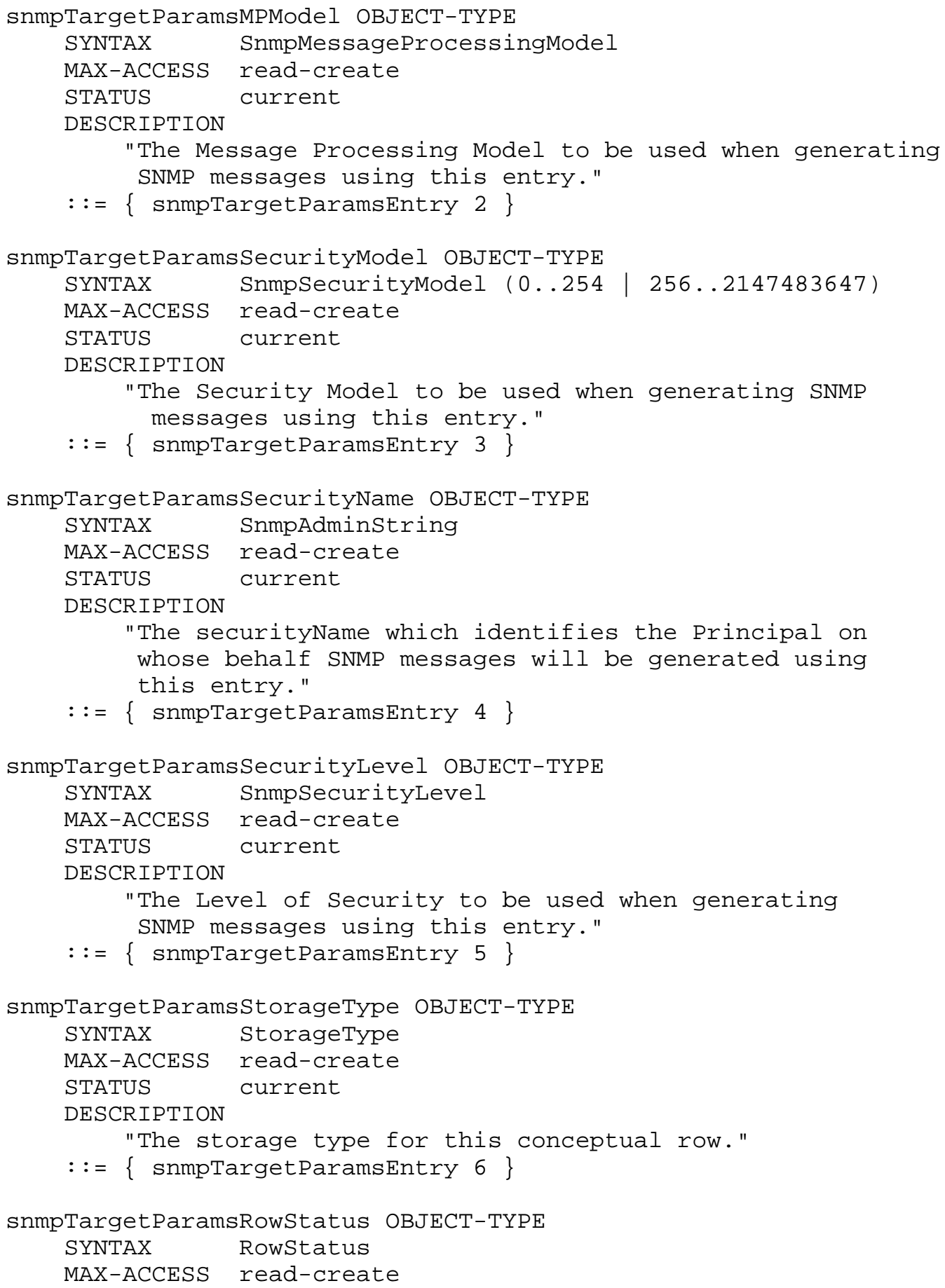




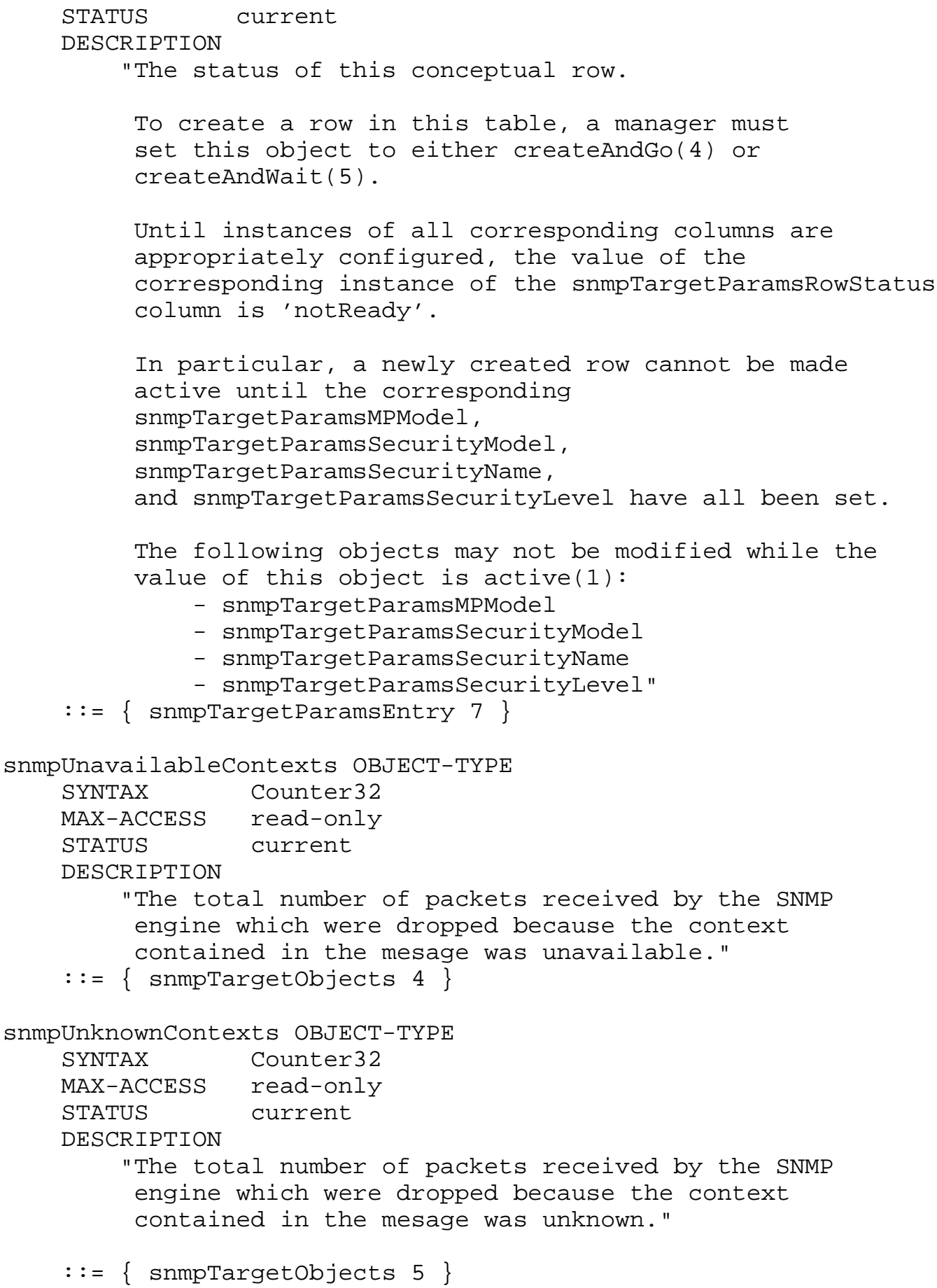




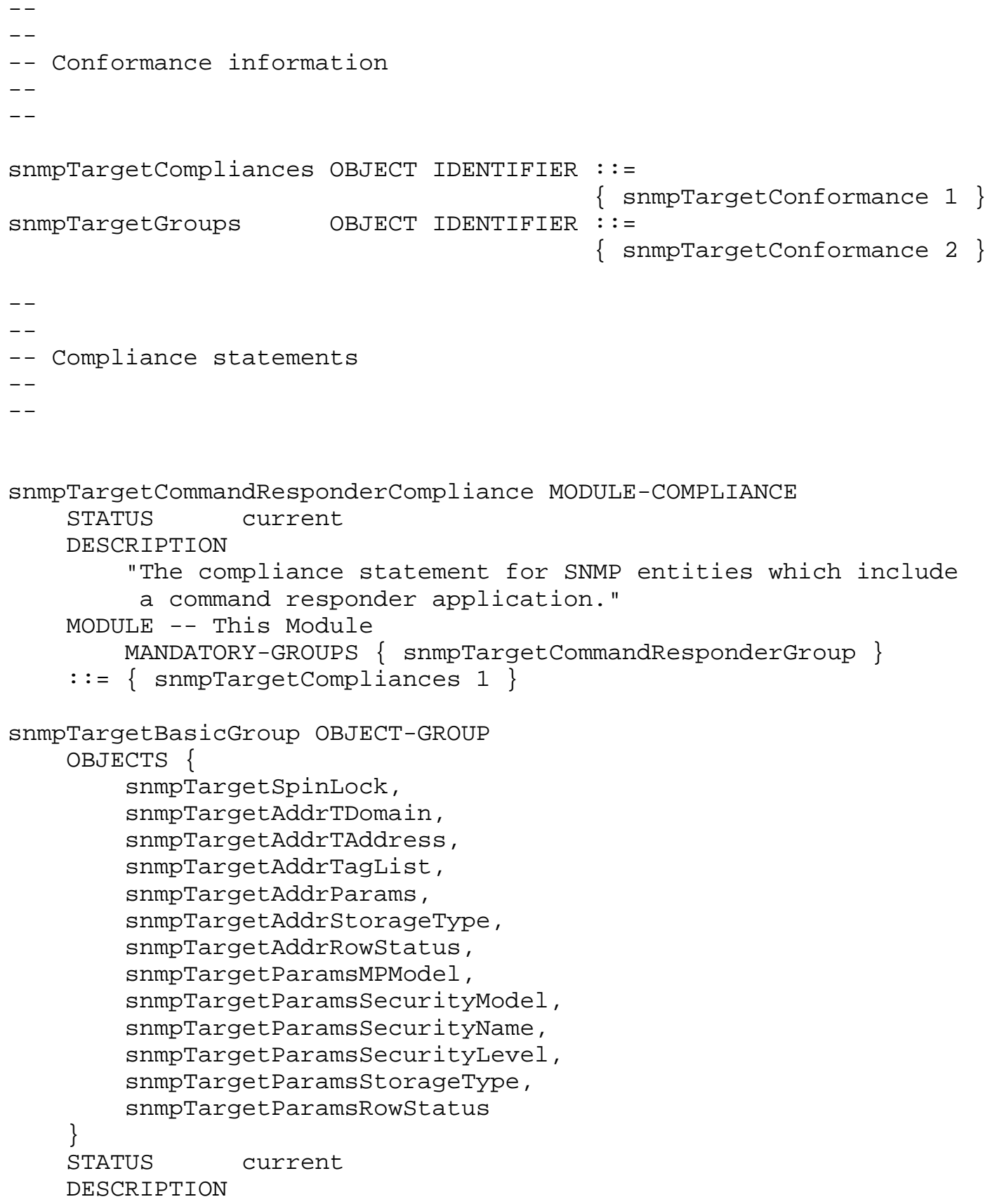

"A collection of objects providing basic remote configuration of management targets." 


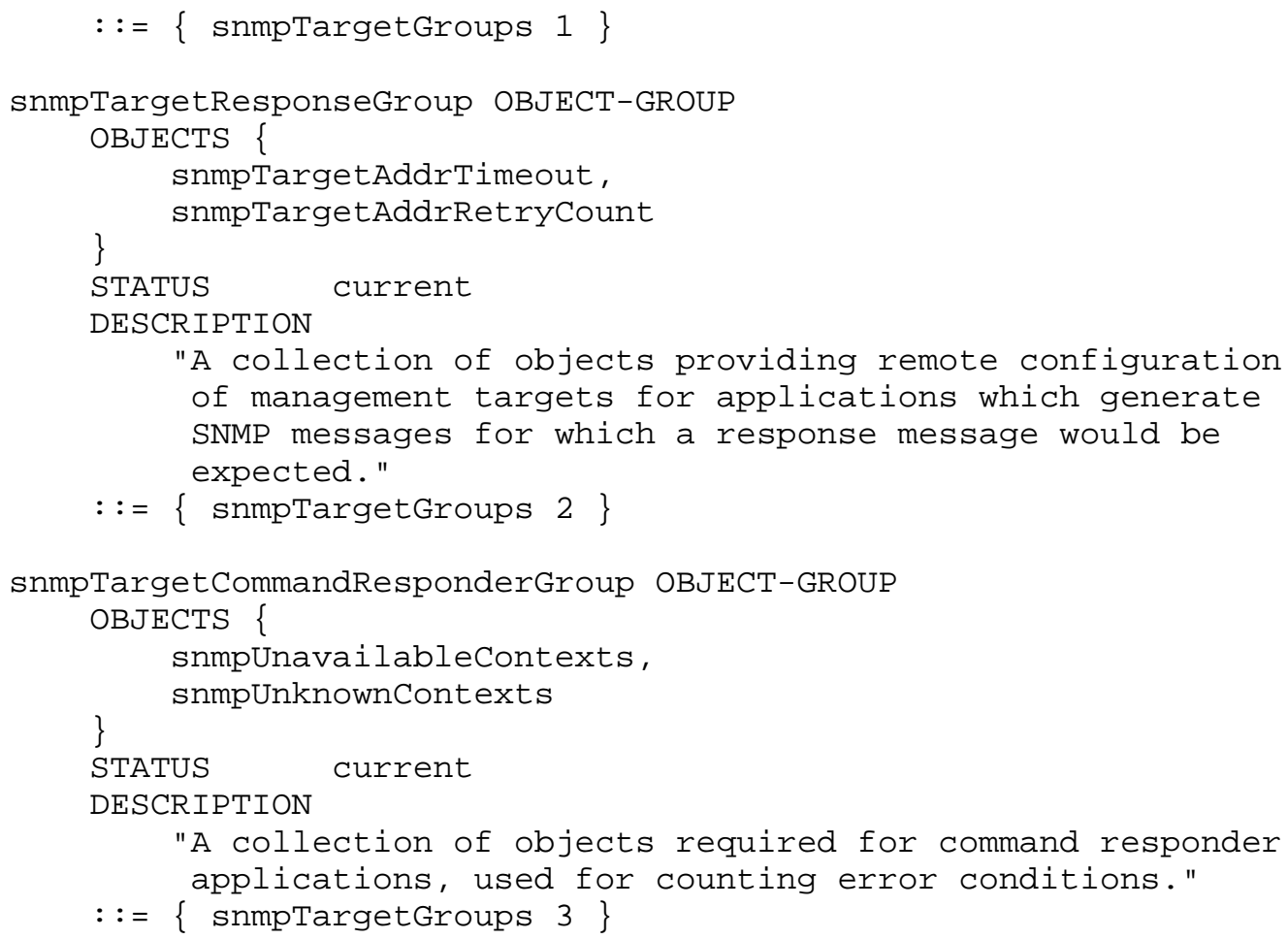

END

\subsection{The Notification MIB Module}

The SNMP-NOTIFICATION-MIB module contains objects for the remote configuration of the parameters used by an SNMP entity for the generation of notifications. It consists of three tables and conformance/compliance statements. The first table, the snmpNotifyTable, contains entries which select which entries in the snmpTargetAddrTable should be used for generating notifications, and the type of notifications to be generated.

The second table sparsely augments the snmpTargetAddrTable with an object which is used to associate a set of filters with a particular management target.

The third table defines filters which are used to limit the number of notifications which are generated using particular management targets. 


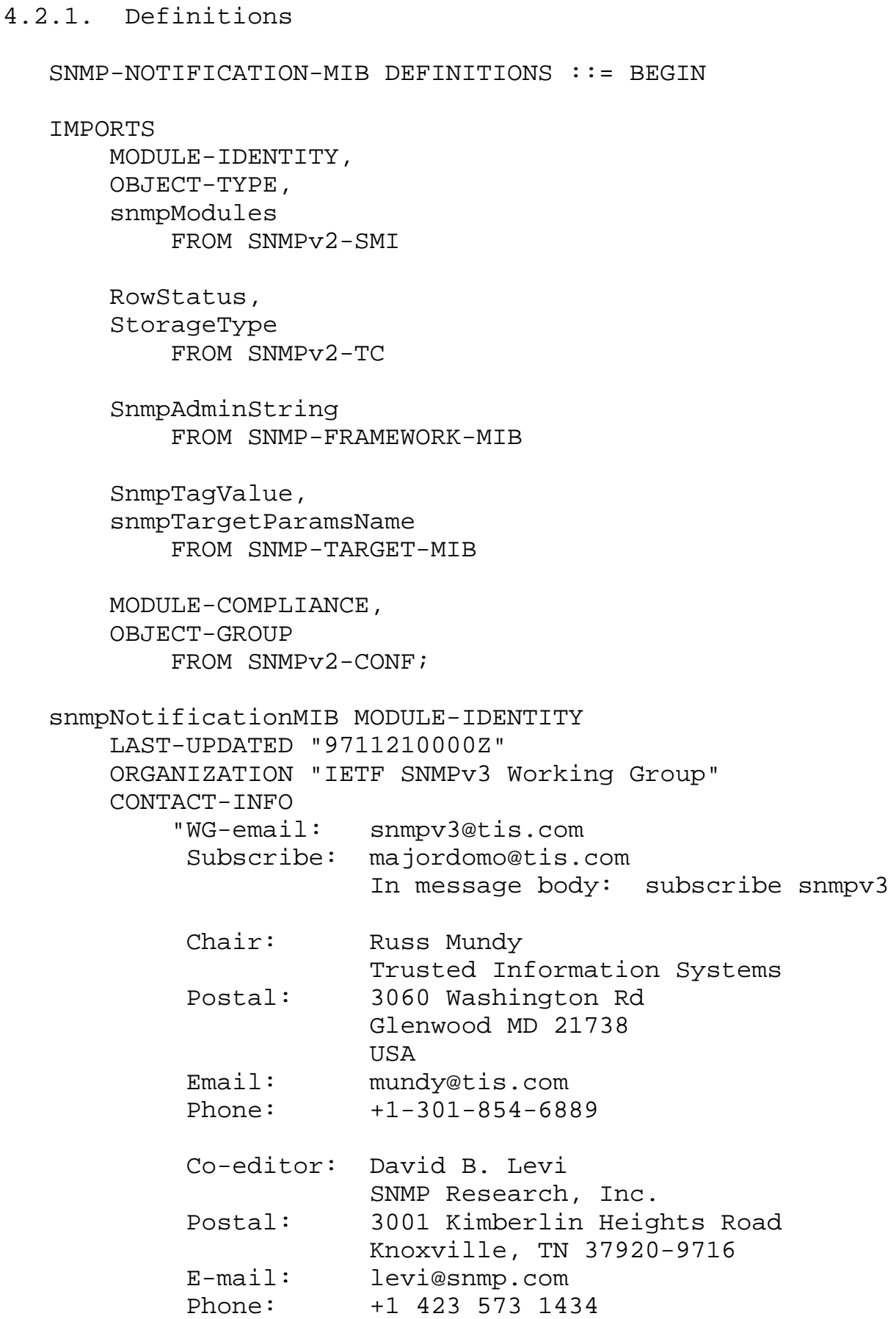




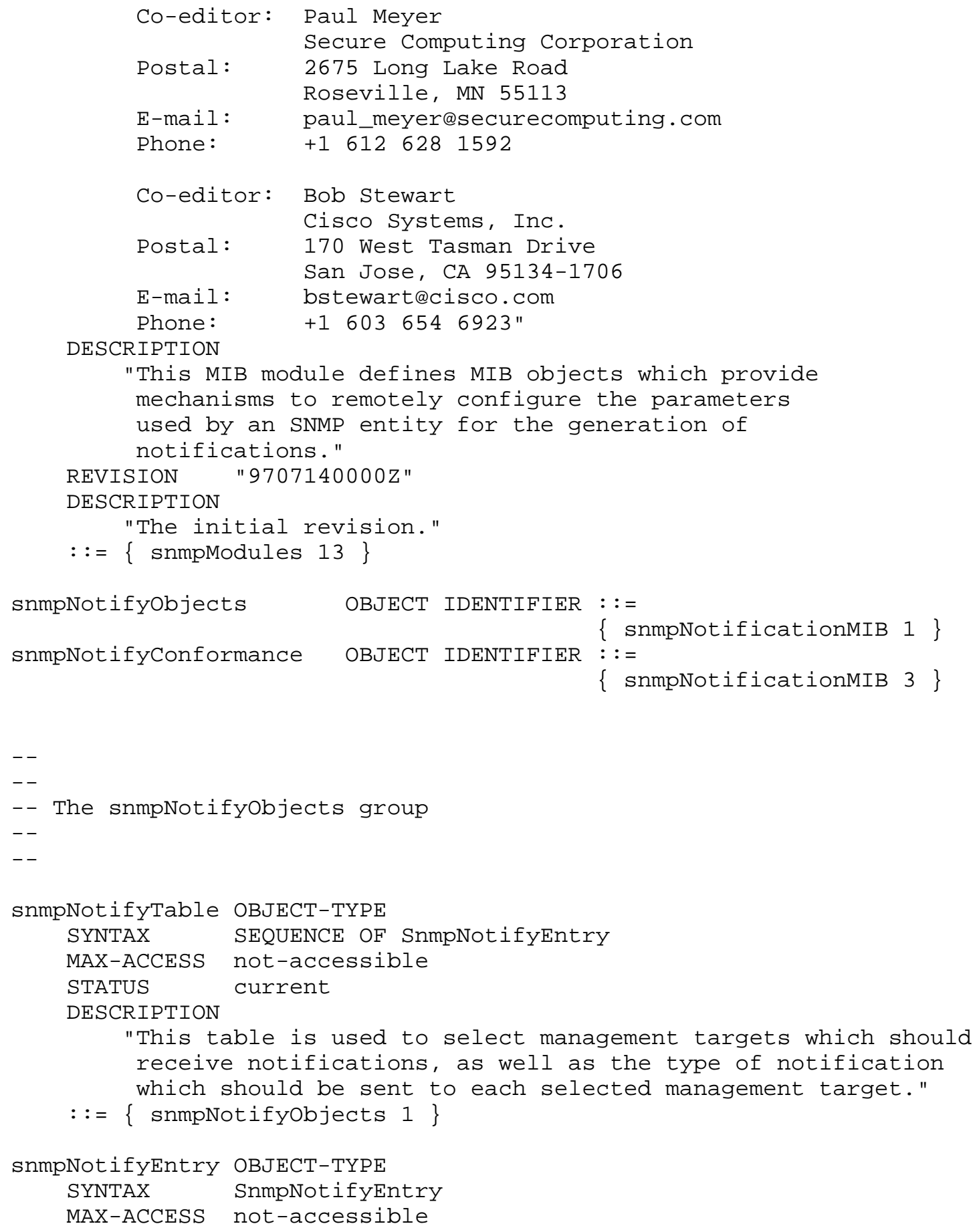




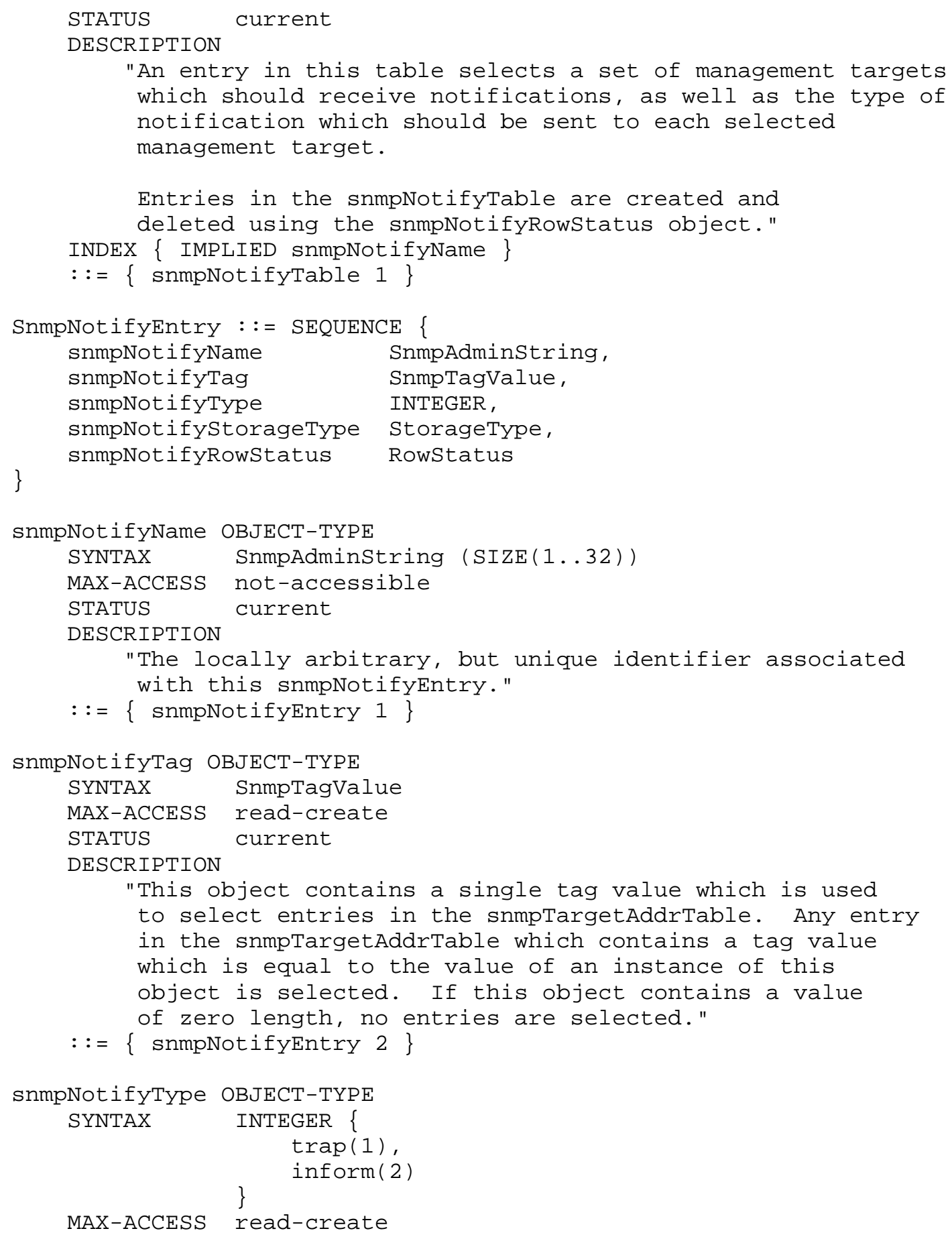




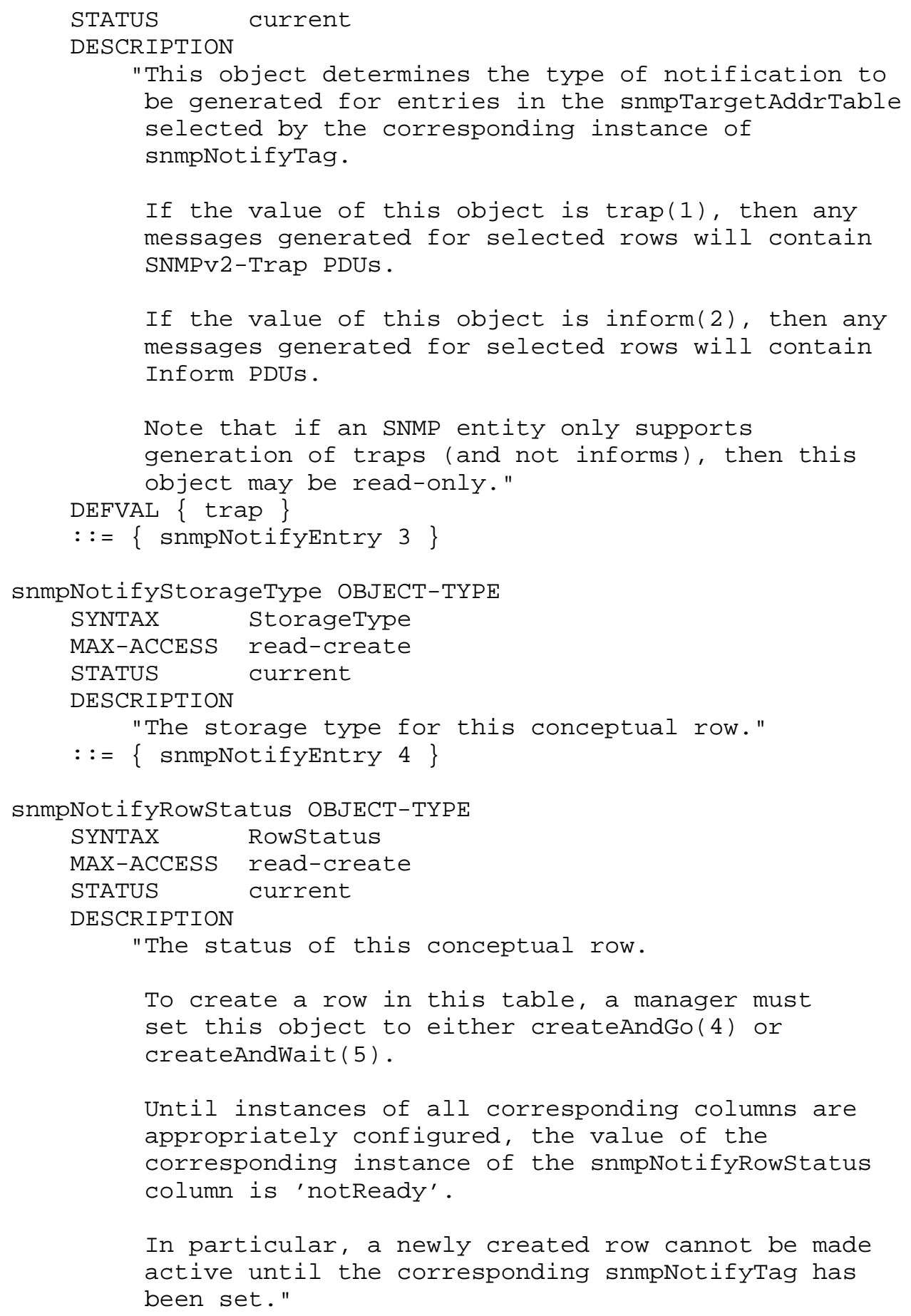


$::=\{$ snmpNotifyEntry 5$\}$

snmpNotifyFilterProfileTable OBJECT-TYPE

SYNTAX SEQUENCE OF SnmpNotifyFilterProfileEntry

MAX-ACCESS not-accessible

STATUS current

DESCRIPTION

"This table is used to associate a notification filter profile with a particular set of target parameters." $::=\{$ snmpNotifyobjects 2$\}$

snmpNotifyFilterProfileEntry OBJECT-TYPE

SYNTAX SnmpNotifyFilterProfileEntry

MAX-ACCESS not-accessible

STATUS current

DESCRIPTION

"An entry in this table indicates the name of the filter profile to be used when generating notifications using the corresponding entry in the snmpTargetParamstable.

Entries in the snmpNotifyFilterProfileTable are created and deleted using the snmpNotifyFilterProfileRowstatus object."

INDEX \{ IMPLIED snmpTargetParamsName \} $::=\{$ snmpNotifyFilterProfileTable 1$\}$

SnmpNotifyFilterProfileEntry : := SEQUENCE \{ snmpNotifyFilterProfileName SnmpAdminstring, snmpNotifyFilterProfilestorType StorageType,

\} snmpNotifyFilterProfileRowStatus RowStatus

snmpNotifyFilterProfilename OBJECT-TYPE

SYNTAX SnmpAdminString (SIZE(1_.32))

MAX-ACCESS read-create

STATUS current

DESCRIPTION

"The name of the filter profile to be used when generating notifications using the corresponding entry in the snmpTargetAddrTable."

$::=\{$ snmpNotifyFilterProfileEntry 1$\}$

snmpNotifyFilterProfilestorType OBJECT-TYPE

SYNTAX StorageType

MAX-ACCESS read-create

STATUS current

DESCRIPTION

"The storage type of this conceptual row." 
$::=\{$ snmpNotifyFilterProfileEntry 2$\}$

snmpNotifyFilterProfileRowstatus OBJECT-TYPE

SYNTAX Rowstatus

MAX-ACCESS read-create

STATUS current

DESCRIPTION

"The status of this conceptual row.

To create a row in this table, a manager must

set this object to either createAndGo(4) or

createAndWait (5)."

$::=\{$ snmpNotifyFilterProfileEntry 3$\}$

snmpNotifyFilterTable OBJECT-TYPE

SYNTAX SEQUENCE OF SnmpNotifyFilterEntry

MAX-ACCESS not-accessible

STATUS current

DESCRIPTION

"The table of filter profiles. Filter profiles are used to determine whether particular management targets should receive particular notifications.

When a notification is generated, it must be compared with the filters associated with each management target which is configured to receive notifications. If the notification is matched by a filter, it is not sent to the management target with which the filter is associated."

$::=\{$ snmpNotifyObjects 3$\}$

snmpNotifyFilterEntry OBJECT-TYPE

SYNTAX SnmpNotifyFilterEntry

MAX-ACCESS not-accessible

STATUS current

DESCRIPTION

"An element of a filter profile.

Entries in the snmpNotifyFilterTable are created and deleted using the snmpNotifyFilterRowstatus object." INDEX \{ snmpNotifyFilterProfileName, IMPLIED snmpNotifyFilterSubtree \} $::=\{$ snmpNotifyFilterTable 1$\}$

SnmpNotifyFilterEntry ::= SEQUENCE \{ snmpNotifyFilterSubtree snmpNotifyFilterMask snmpNotifyFilterType

OBJECT IDENTIFIER, OCTET STRING, INTEGER, 


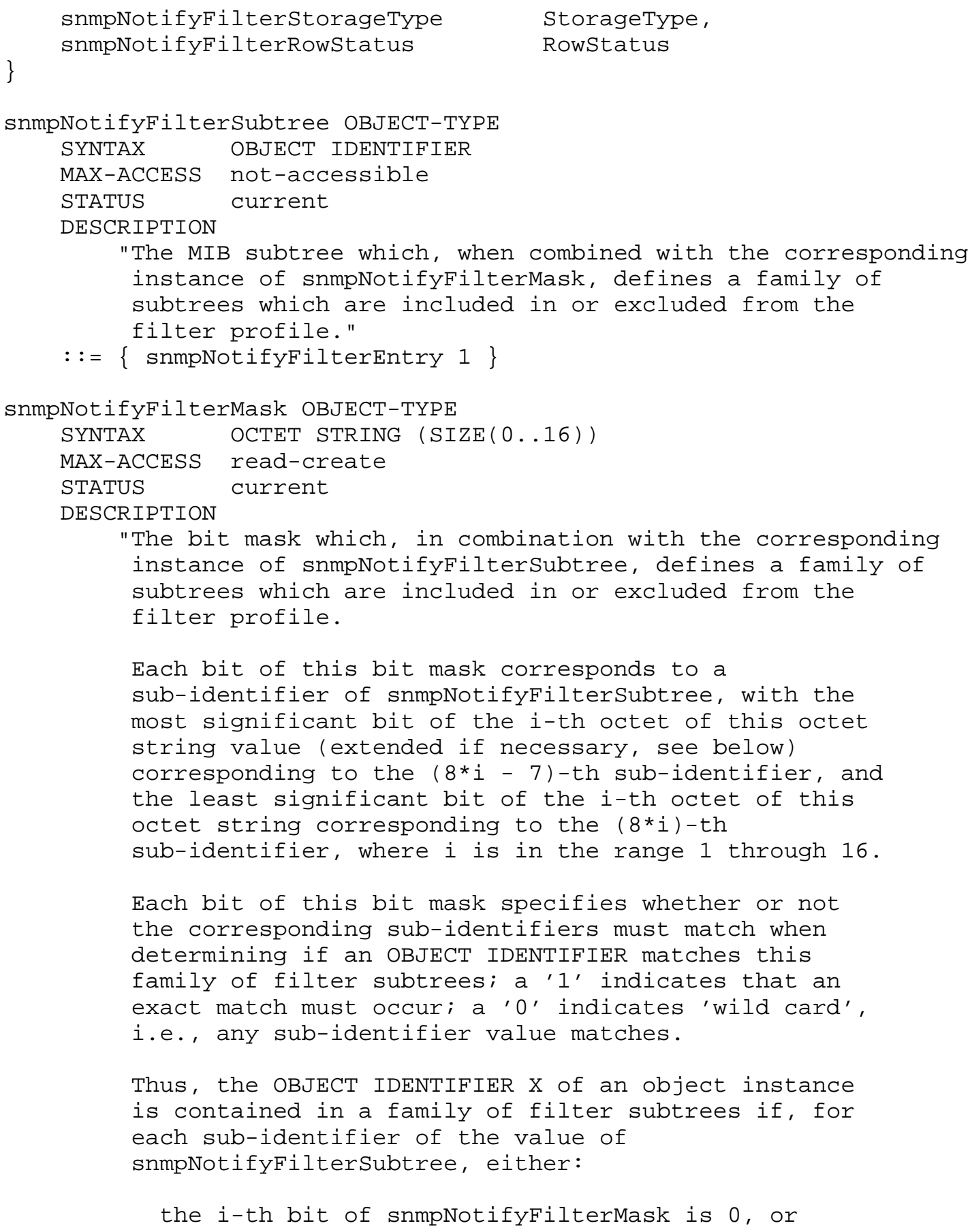


the i-th sub-identifier of $x$ is equal to the $i-t h$ sub-identifier of the value of snmpNotifyFilterSubtree.

If the value of this bit mask is M bits long and there are more than $M$ sub-identifiers in the corresponding instance of snmpNotifyFilterSubtree, then the bit mask is extended with $1^{\prime} \mathrm{s}$ to be the required length.

Note that when the value of this object is the zero-length string, this extension rule results in a mask of all-1's being used (i.e., no 'wild card'), and the family of filter subtrees is the one subtree uniquely identified by the corresponding instance of snmpNotifyFilterSubtree."

DEFVAL $\left\{\prime^{\prime}\right.$ ' $\left.\mathrm{H}\right\}$

$::=\{$ snmpNotifyFilterEntry 2$\}$

snmpNotifyFilterType OBJECT-TYPE

SYNTAX INTEGER \{ included (1), excluded (2)

\}

MAX-ACCESS read-create

STATUS current

DESCRIPTION

"This object indicates whether the family of filter subtrees defined by this entry are included in or excluded from a filter."

DEFVAL \{ included \}

$::=\{$ snmpNotifyFilterEntry 3$\}$

snmpNotifyFilterStorageType OBJECT-TYPE

SYNTAX StorageType

MAX-ACCESS read-create

STATUS current

DESCRIPTION

"The storage type of this conceptual row." $::=\{$ snmpNotifyFilterEntry 4$\}$

snmpNotifyFilterRowStatus OBJECT-TYPE

SYNTAX Rowstatus

MAX-ACCESS read-create

STATUS current

DESCRIPTION

"The status of this conceptual row. 


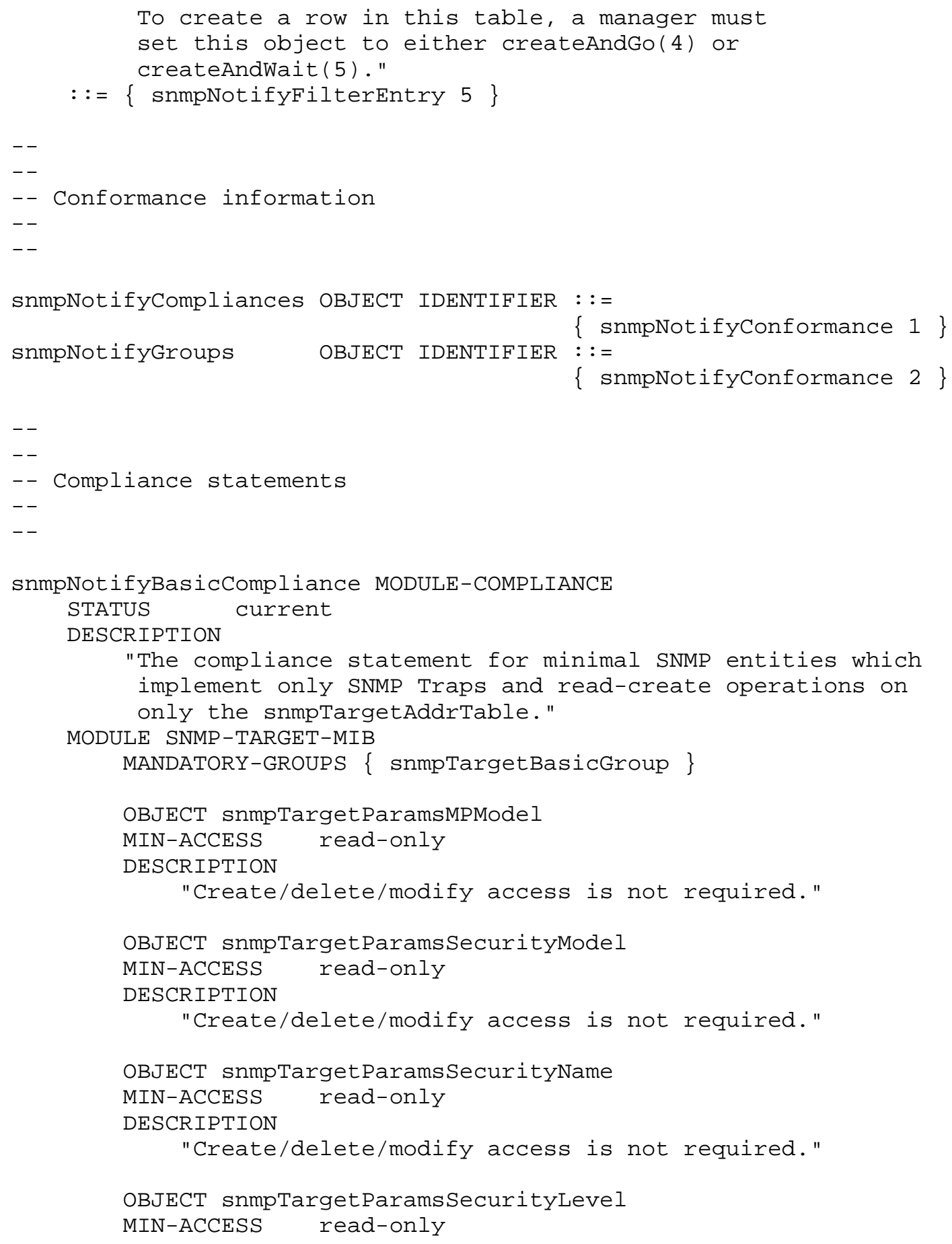




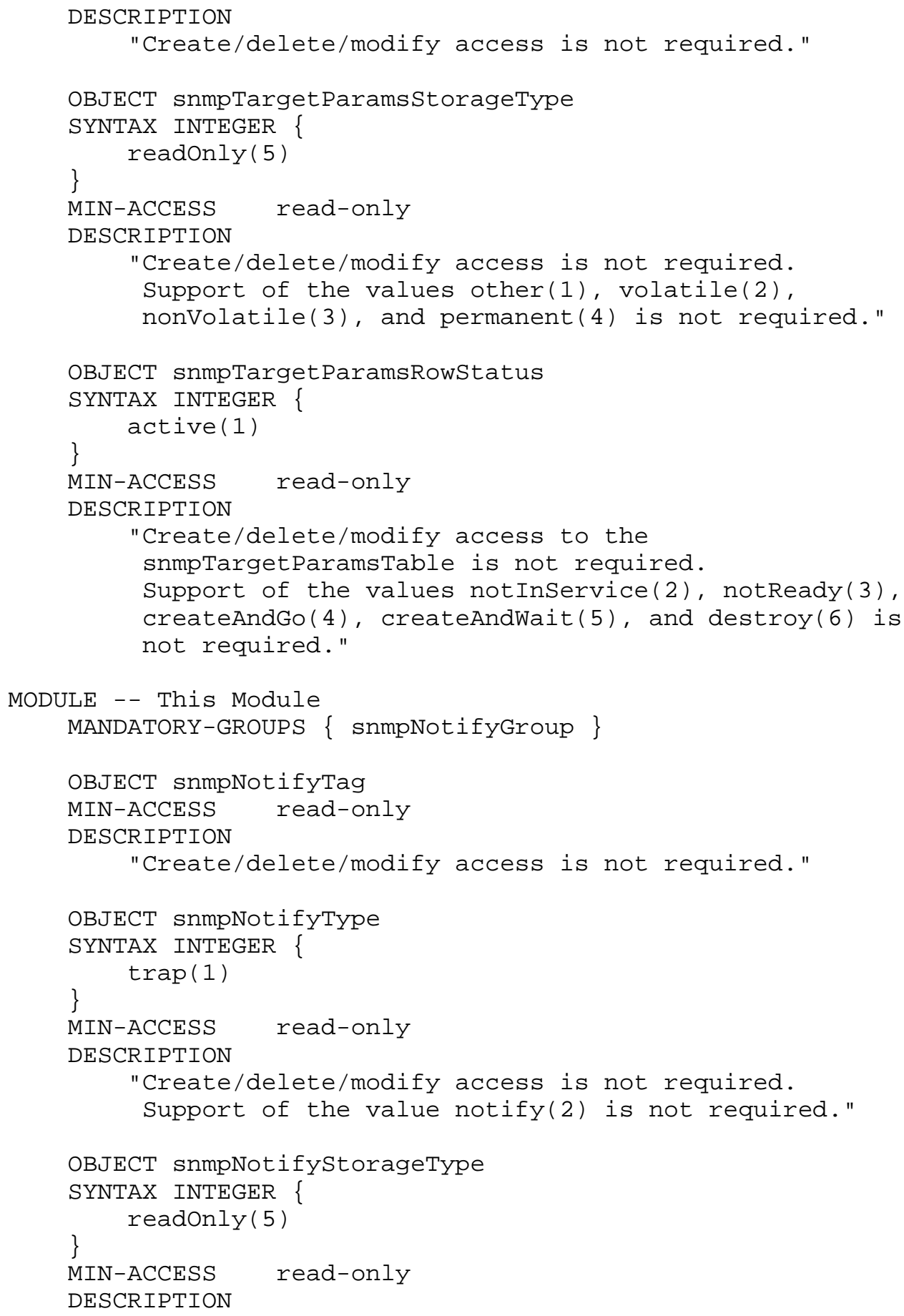




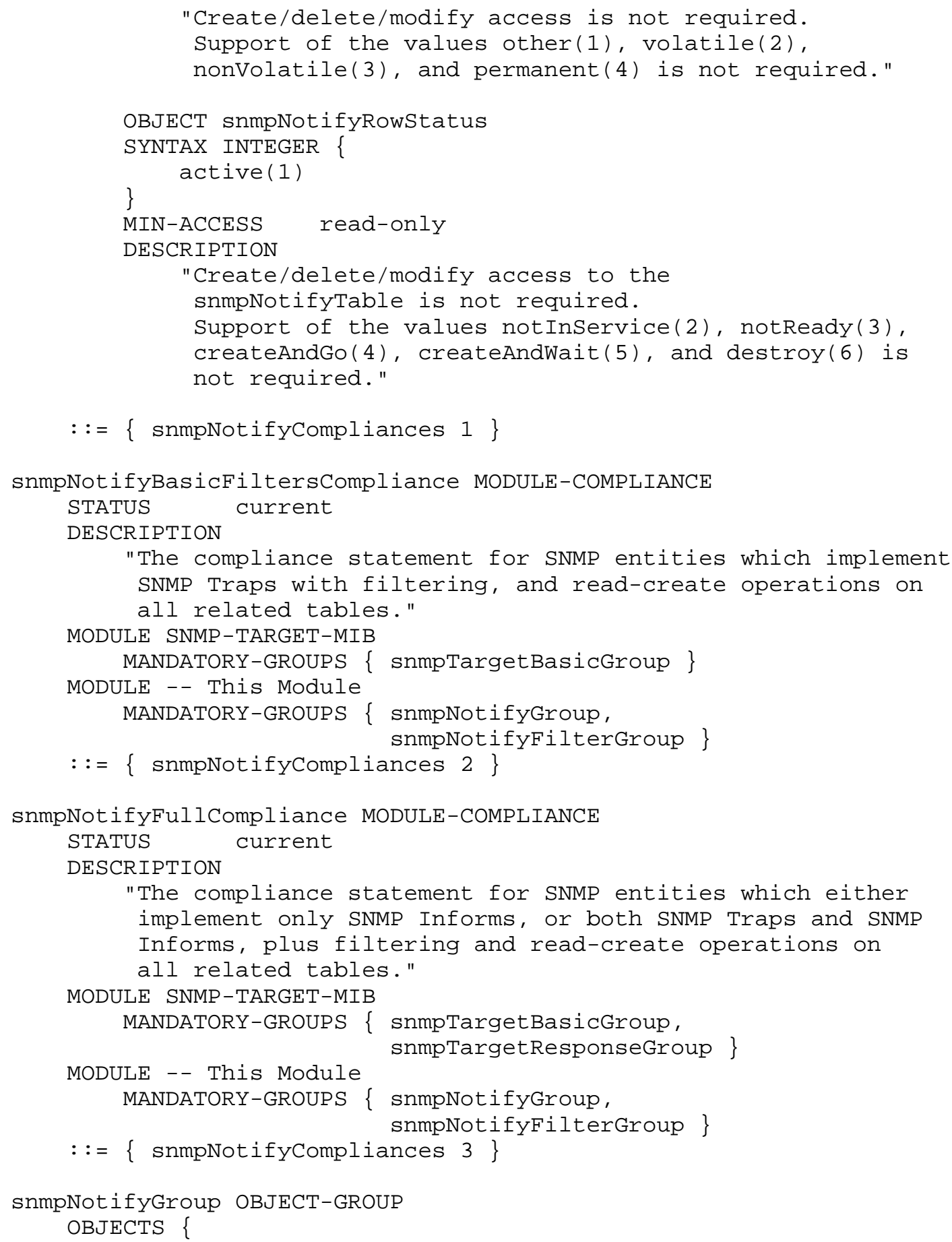




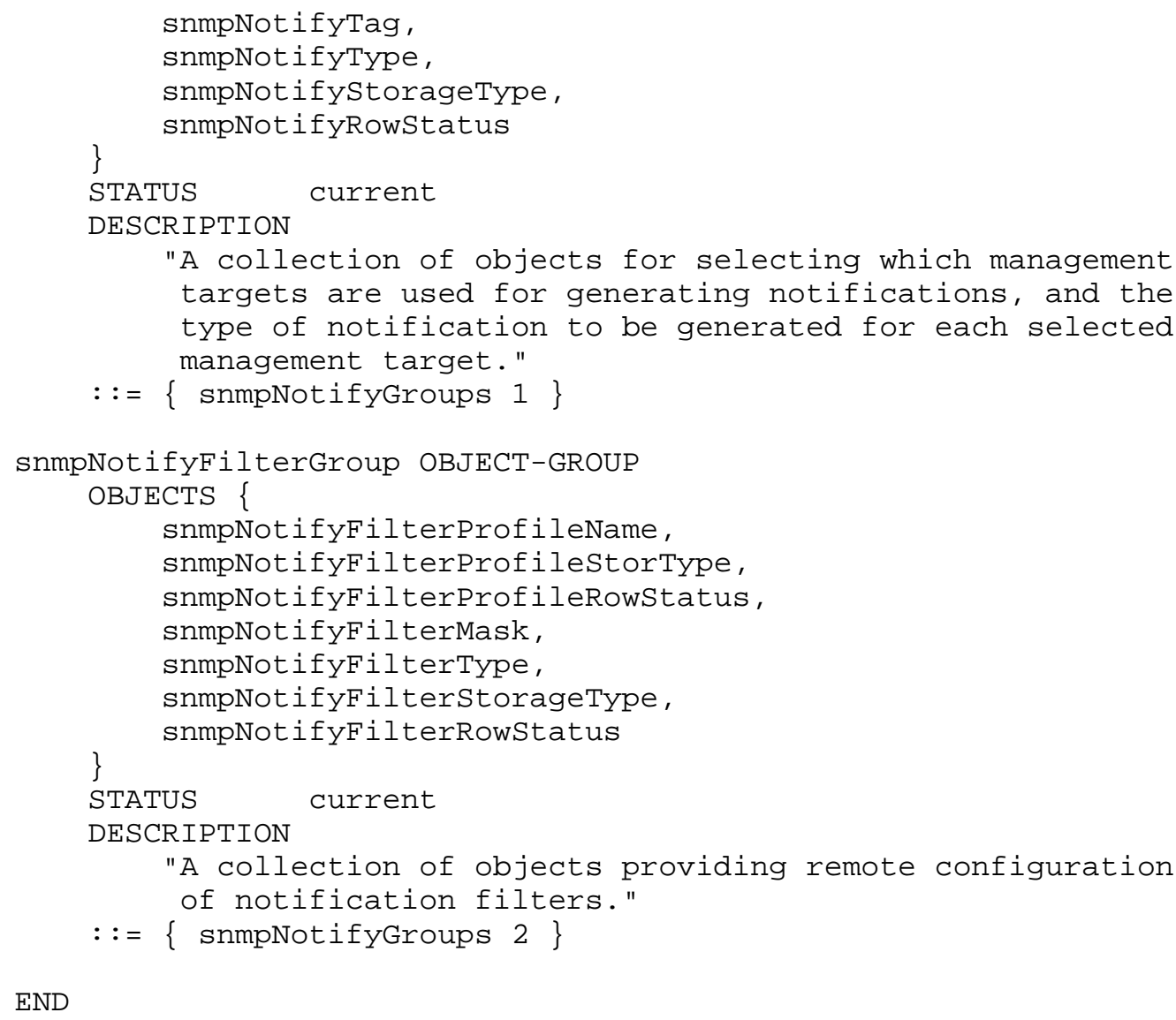

END

\subsection{The Proxy MIB Module}

The SNMP-PROXY-MIB module, which defines MIB objects that provide mechanisms to remotely configure the parameters used by an SNMP entity for proxy forwarding operations, contains a single table. This table, snmpproxyTable, is used to define translations between management targets for use when forwarding messages.

4.3.1. Definitions

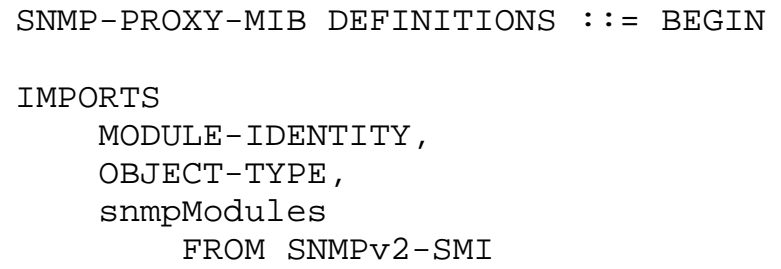




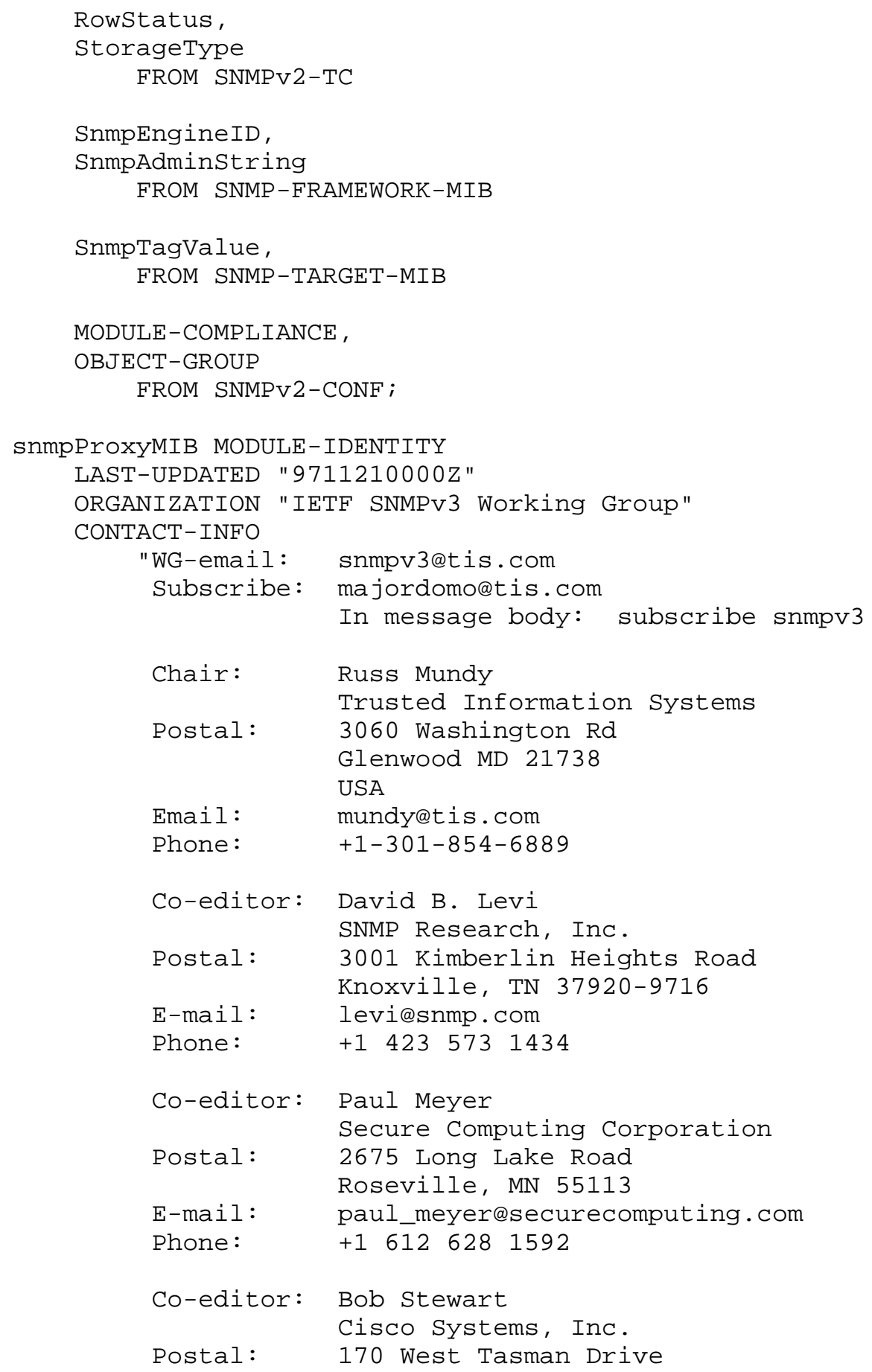




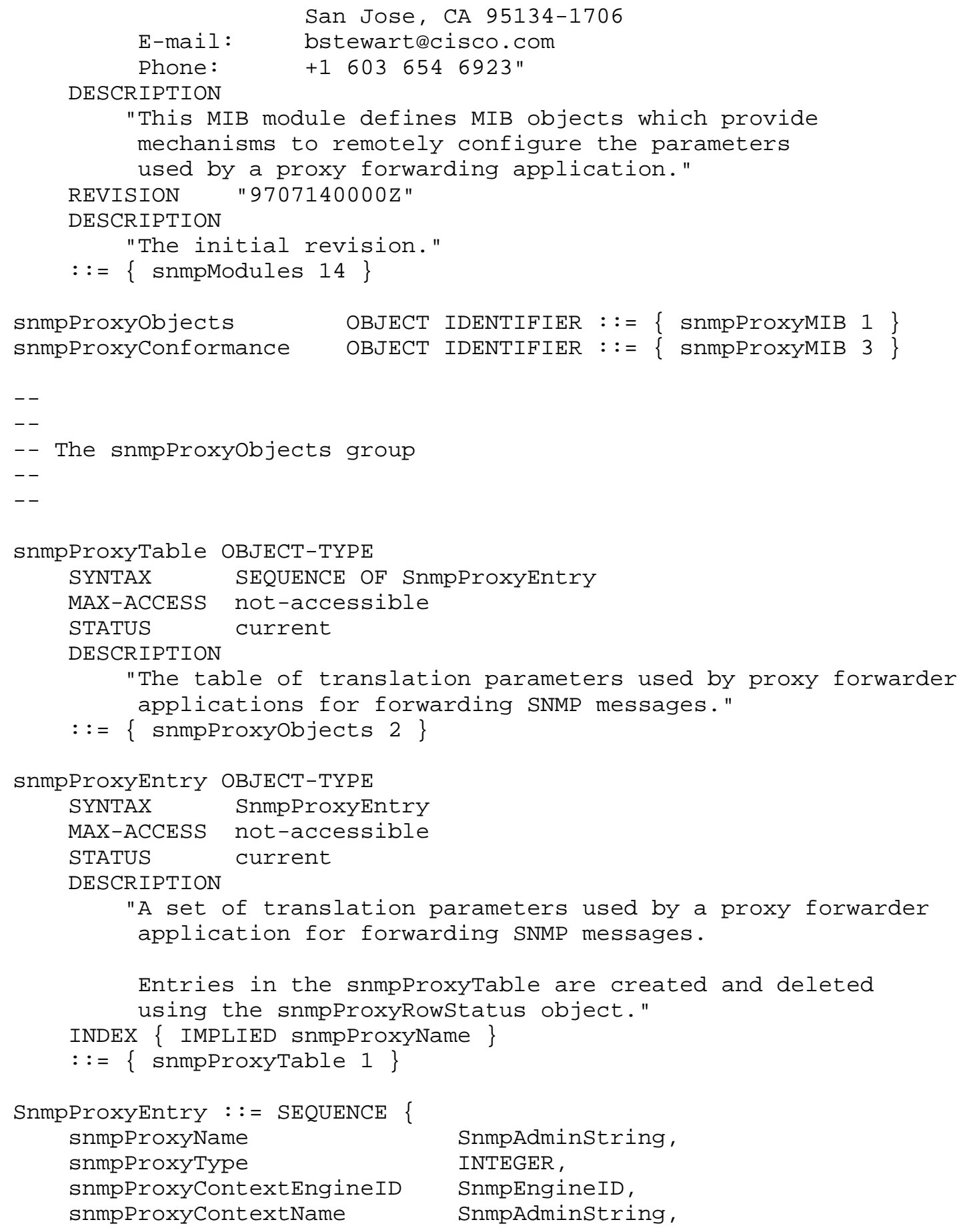




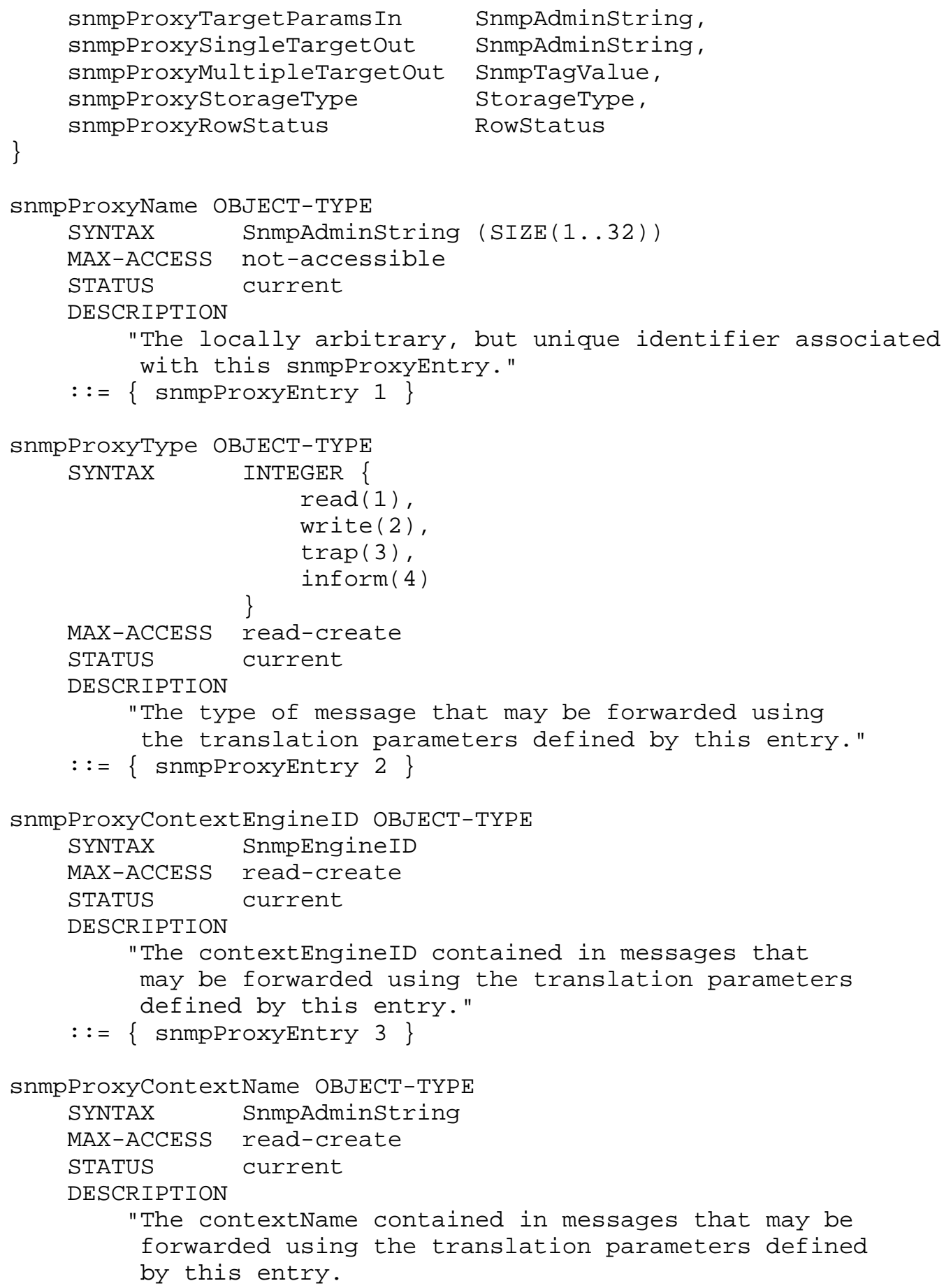


This object is optional, and if not supported, the contextName contained in a message is ignored when selecting an entry in the snmpProxyTable."

$::=\{$ snmpProxyEntry 4$\}$

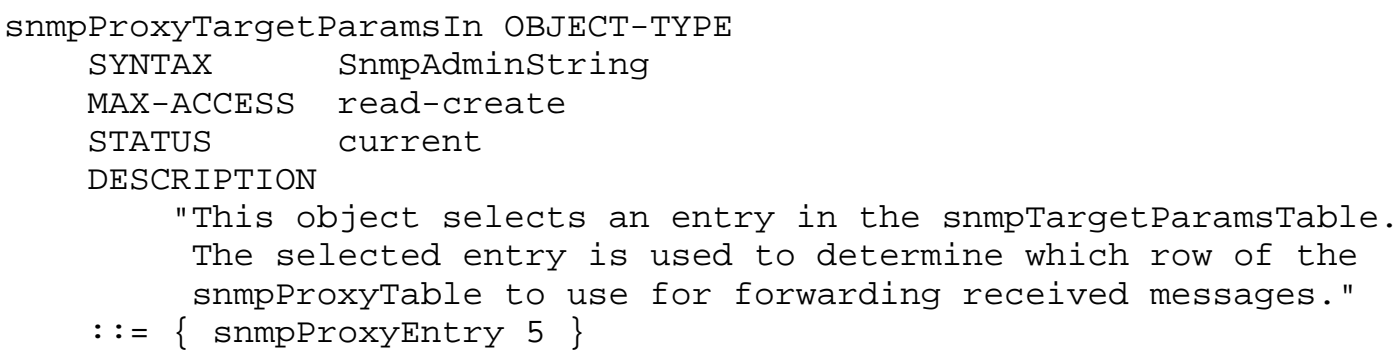




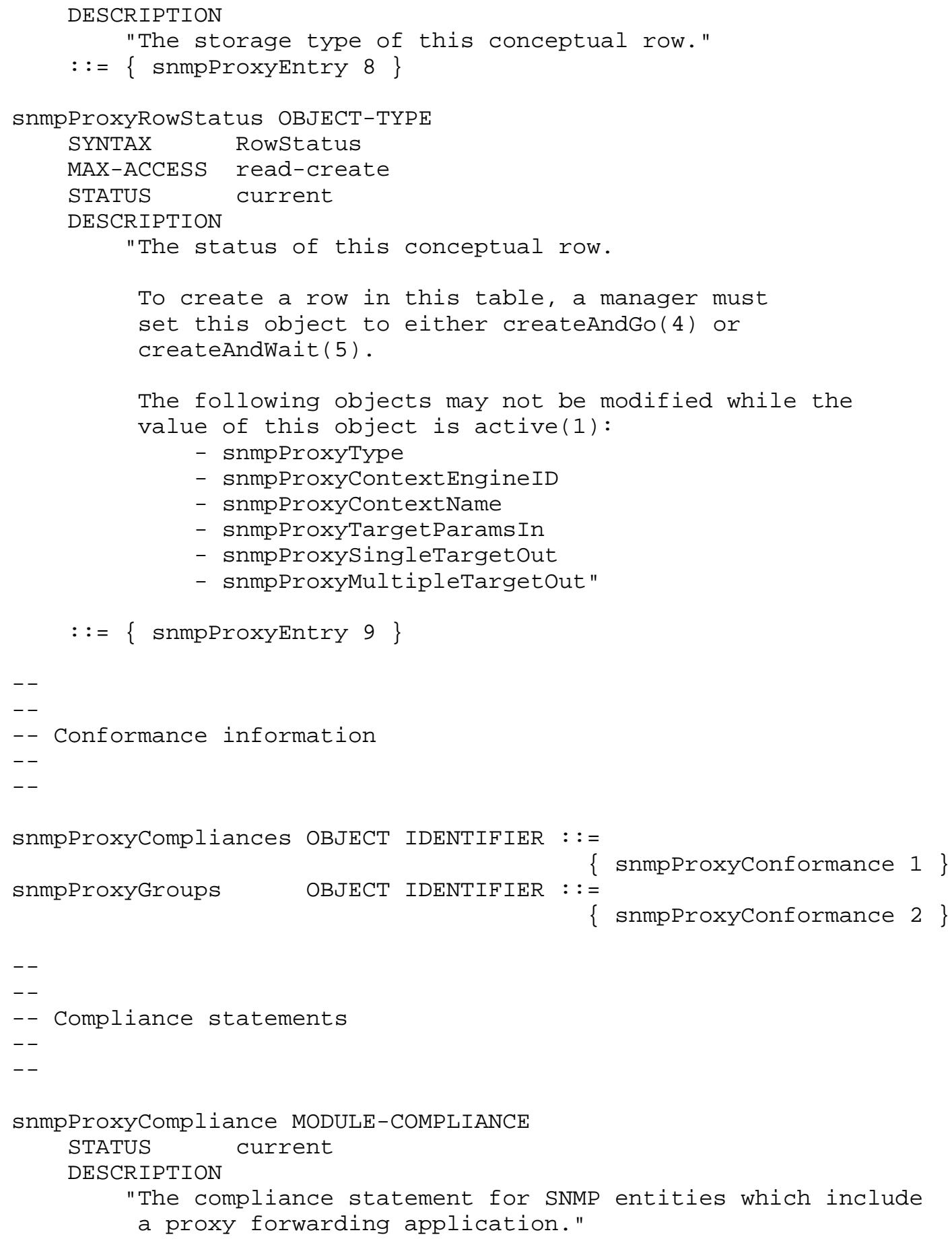




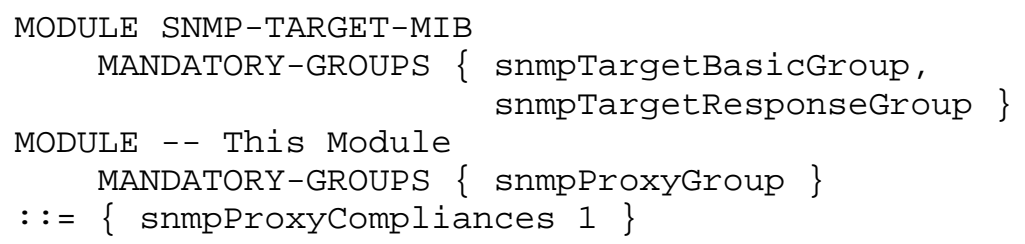

END

5. Identification of Management Targets in Notification Originators

This section describes the mechanisms used by a notification originator application when using the MIB module described in this document to determine the set of management targets to be used when generating a notification.

A notification originator uses the snmpNotifyTable to find the management targets to be used for generating notifications. Each active entry in this table identifies zero or more entries in the snmpTargetAddrTable. Any entry in the snmpTargetAddrTable whose snmpTargetAddrTagList object contains a tag value which is equal to a value of snmpNotifyTag is selected by the snmpNotifyEntry which contains that instance of snmpNotifyTag. Note that a particular snmpTargetAddrEntry may be selected by multiple entries in the snmpNotifyTable, resulting in multiple notifications being generated using that snmpTargetAddrEntry. 
Each snmpTargetAddrEntry contains a pointer to the snmpTargetParamsTable (snmpTargetAddrParams). This pointer selects a set of SNMP parameters to be used for generating notifications. If the selected entry in the snmpTargetParamsTable does not exist, the management target is not used to generate notifications.

The decision as to whether a notification should contain an SNMPv2Trap or Inform PDU is determined by the value of the snmpNotifyType object. If the value of this object is trap(1), the notification should contain an SNMPV2-Trap PDU. If the value of this object is inform(2), then the notification should contain an Inform PDU, and the timeout time and number of retries for the Inform are the value of snmpTargetAddrTimeout and snmpTargetAddrRetryCount. Note that the exception to these rules is when the snmpTargetParamsMPModel object indicates SNMPV1. In this case, the notification is sent as a Trap if the value of snmpNotifyTargetType is either trap(1) or inform(2).

6. Notification Filtering

This section describes the mechanisms used by a notification originator application when using the MIB module described in this document to filter generation of notifications.

A notification originator uses the snmpNotifyFilterTable to filter notifications. A notification filter profile may be associated with a particular entry in the snmpTargetparamsTable. The associated filter profile is identified by an entry in the snmpNotifyFilterProfiletable whose index is equal to the index of the entry in the snmptargetparamsTable. If no such entry exists in the snmpNotifyFilterProfileTable, no filtering is performed for that management target.

If such an entry does exist, the value of snmpNotifyFilterProfileName of the entry is compared with the corresponding portion of the index of all active entries in the snmpNotifyfilterTable. All such entries for which this comparison results in an exact match are used for filtering a notification generated using the associated snmpTargetparamsEntry. If no such entries exist, no filtering is performed, and a notification may be sent to the management target.

Otherwise, if matching entries do exist, a notification may be sent if the NOTIFICATION-TYPE OBJECT IDENTIFIER of the notification (this is the value of the element of the variable bindings whose name is snmpTrapoID.0, i.e., the second variable binding), and all of the object instances to be included in the variable-bindings of the notification, are not specifically excluded by the matching entries. 
Each set of snmpNotifyFilterTable entries is divided into two collections of filter subtrees: the included filter subtrees, and the excluded filter subtrees. The snmpNotifyFilterType object defines the collection to which each matching entry belongs.

To determine whether a particular notification name or object instance is excluded by the set of matching entries, compare the notification name's or object instance's OBJECT IDENTIFIER with each of the matching entries. If none match, then the notification name or object instance is considered excluded, and the notification should not be sent to this management target. If one or more match, then the notification name or object instance is included or excluded, according to the value of snmpNotifyFilterType in the entry whose value of snmpNotifyFiltersubtree has the most sub-identifiers. If multiple entries match and have the same number of subidentifiers, then the lexicographically greatest instance of snmpNotifyFilterType among those which match determines the inclusion or exclusion.

A notification name's or object instance's OBJECT IDENTIFIER $\mathrm{X}$ matches an entry in the snmpNotifyFilterTable when the number of sub-identifiers in $X$ is at least as many as in the value of snmpNotifyFilterSubtree for the entry, and each sub-identifier in the value of snmpNotifyFilterSubtree matches its corresponding subidentifier in $X$. Two sub-identifiers match either if the corresponding bit of snmpNotifyFilterMask is zero (the 'wild card' value), or if the two sub-identifiers are equal.

7. Management Target Translation in Proxy Forwarder Applications

This section describes the mechanisms used by a proxy forwarder application when using the MIB module described in this document to translate incoming management target information into outgoing management target information for the purpose of forwarding messages. There are actually two mechanisms a proxy forwarder may use, one for forwarding request messages, and one for forwarding notification messages.

\subsection{Management Target Translation for Request Forwarding}

When forwarding request messages, the proxy forwarder will select a single entry in the snmpproxyTable. To select this entry, it will perform the following comparisons:

- The snmpproxyType must be read(1) if the request is a Get, GetNext, or GetBulk request. The snmpProxyType must be write(2) if the request is a set request. 
- The contextEngineId must equal the snmpProxyContextEngineID object.

- If the snmpProxyContextName object is supported, it must equal the context Name.

- The snmpProxyTargetParamsIn object identifies an entry in the snmpTargetParamsTable. The messageProcessingModel, securitylevel, security model, and securityName must match the values of snmpTargetParamsMPModel, snmpTargetParamsSecurityModel, snmpTargetParamsSecurityName, and snmptargetParams Securitylevel of the identified entry in the snmpTargetParamsTable.

There may be multiple entries in the snmpProxyTable for which these comparisons succeed. The entry whose snmpProxyName has the lexicographically smallest value and for which the comparisons succeed will be selected by the proxy forwarder.

The outgoing management target information is identified by the value of the snmpProxySingleTargetout object of the selected entry. This object identifies an entry in the snmpTargetAddrTable. The identified entry in the snmpTargetAddrTable also contains a reference to the snmpTargetParamsTable (snmpTargetAddrParams). If either the identified entry in the snmpTargetAddrTable does not exist, or the identified entry in the snmpTargetParamsTable does not exist, then this snmpProxyEntry does not identify valid forwarding information, and the proxy forwarder should attempt to identify another row.

If there is no entry in the snmpProxyTable for which all of the conditions above may be met, then there is no appropriate forwarding information, and the proxy forwarder should take appropriate actions.

Otherwise, The snmpTargetAddrTDomain, snmpTargetAddrTAddress, snmpTargetAddrTimeout, and snmpTargetRetryCount of the identified snmpTargetAddrEntry, and the snmpTargetParamsMPModel, snmpTargetParams SecurityModel, snmpTargetParamsSecurityName, and snmpTargetParamsSecurityLevel of the identified snmpTargetParamsEntry are used as the destination management target.

\subsection{Management Target Translation for Notification Forwarding}

When forwarding notification messages, the proxy forwarder will select multiple entries in the snmpProxyTable. To select these entries, it will perform the following comparisons: 
- The snmproxyType must be trap(3) if the notification is a Trap. The snmpProxytype must be inform(4) if the request is an Inform.

- The contextEngineId must equal the snmpProxyContextEngineID object.

- If the snmpProxyContextName object is supported, it must equal the contextName.

- The snmpproxyTargetParamsIn object identifies an entry in the snmpTargetParamsTable. The messageProcessingModel, securitylevel, security model, and securityName must match the values of snmpTargetParamsMPModel, snmpTargetParamsSecurityModel, snmpTargetParamsSecurityName, and snmptargetParams Securitylevel of the identified entry in the snmpTargetParamsTable.

All entries for which these conditions are met are selected. The snmpProxyMultipleTargetout object of each such entry is used to select a set of entries in the snmpTargetAddrTable. Any snmpTargetAddrEntry whose snmpTargetAddrTagList object contains a tag value equal to the value of snmpProxyMultipleTargetout, and whose snmpTargetAddrParams object references an existing entry in the snmpTargetParamstable, is selected as a destination for the forwarded notification.

8. Intellectual Property

The IETF takes no position regarding the validity or scope of any intellectual property or other rights that might be claimed to pertain to the implementation or use of the technology described in this document or the extent to which any license under such rights might or might not be available; neither does it represent that it has made any effort to identify any such rights. Information on the IETF's procedures with respect to rights in standards-track and standards-related documentation can be found in BCP-11. Copies of claims of rights made available for publication and any assurances of licenses to be made available, or the result of an attempt made to obtain a general license or permission for the use of such proprietary rights by implementors or users of this specification can be obtained from the IETF Secretariat.

The IETF invites any interested party to bring to its attention any copyrights, patents or patent applications, or other proprietary rights which may cover technology that may be required to practice this standard. Please address the information to the IETF Executive Director. 


\section{Acknowledgments}

This document is the result of the efforts of the SNMPv3 Working Group. Some special thanks are in order to the following SNMPv3 WG members :

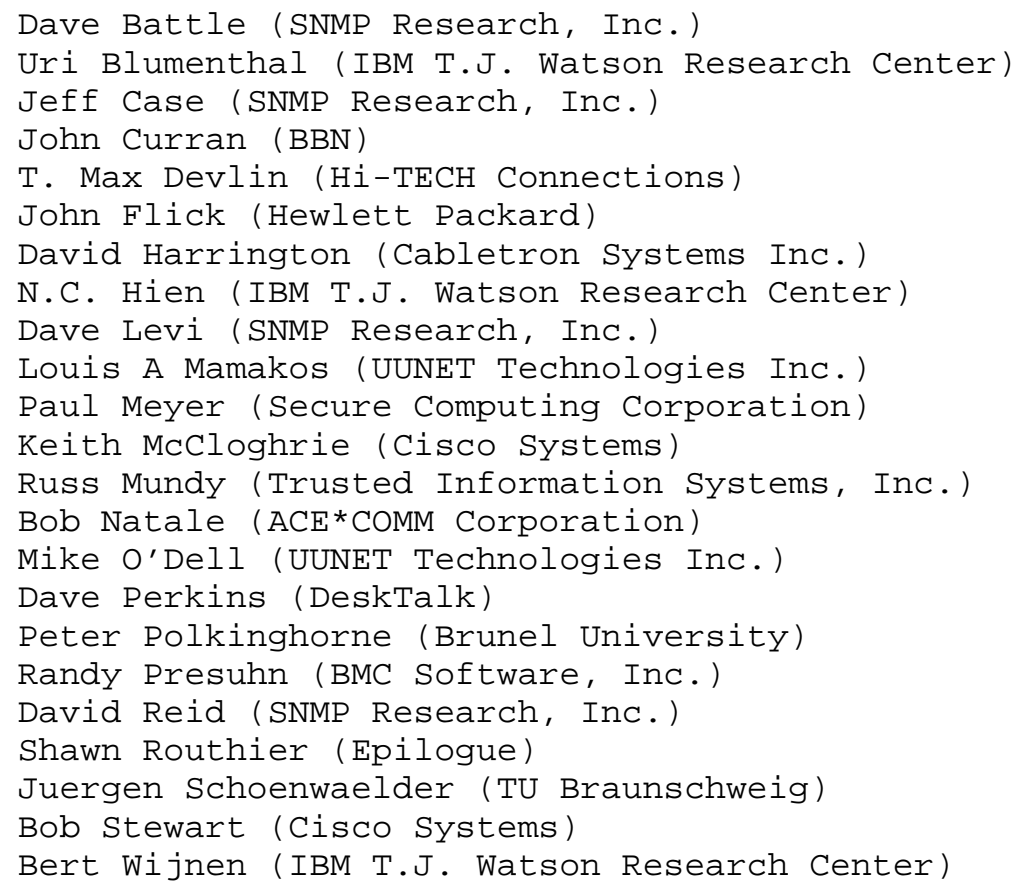




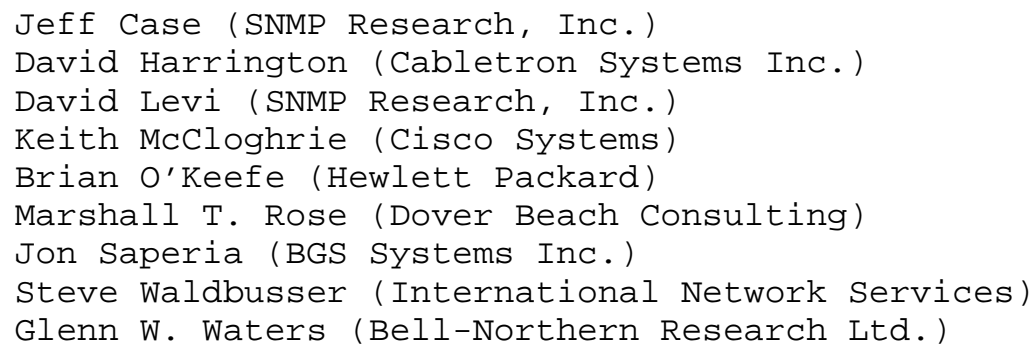

10. Security Considerations

The SNMP applications described in this document typically have direct access to MIB instrumentation. Thus, it is very important that these applications be strict in their application of access control as described in this document.

In addition, there may be some types of notification generator applications which, rather than accessing MIB instrumentation using access control, will obtain MIB information through other means (such as from a command line). The implementors and users of such applications must be responsible for not divulging MIB information that normally would be inaccessible due to access control.

11. References

[RFC1157]

Case, J., Fedor, M., Schoffstall, M. and J. Davin, "Simple Network Management Protocol", RFC 1157, May 1990.

[RFC1213]

McCloghrie, K. and M. Rose, Editors, "Management Information Base for Network Management of TCP/IP-based internets: MIB-II", STD 17, RFC 1213, March 1991.

[RFC1902]

Case, J., McCloghrie, K., Rose, M. and S. Waldbusser, "Structure of Management Information for Version 2 of the Simple Network Management Protocol (SNMPv2)", RFC 1902, January 1996.

[RFC1903]

Case, J., McCloghrie, K., Rose, M. and S. Waldbusser, "Textual Conventions for Version 2 of the Simple Network Management Protocol (SNMPV2)", RFC 1903, January 1996. 


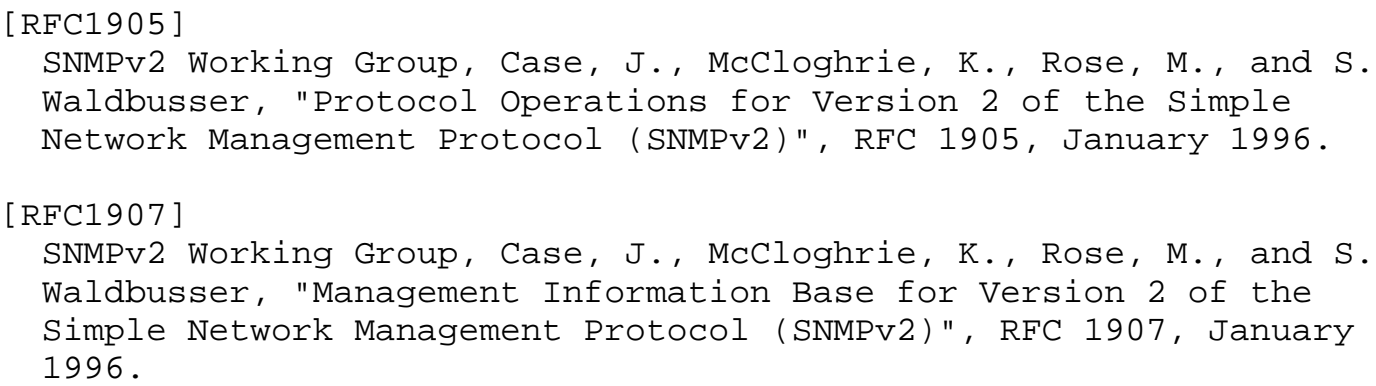




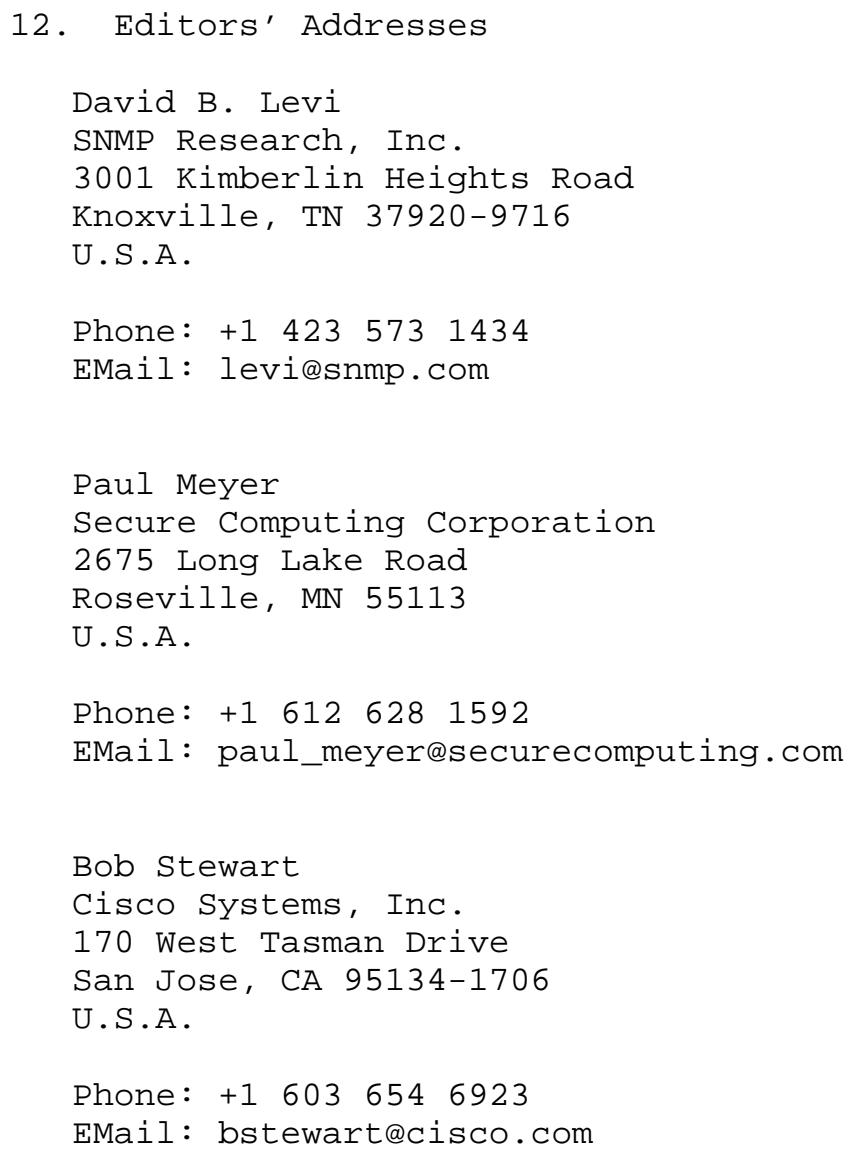


APPENDIX A - Trap Configuration Example

This section describes an example configuration for a Notification Generator application which implements the snmpNotifyBasicCompliance level. The example configuration specifies that the Notification Generator should send notifications to 3 separate managers, using authentication and no privacy for the first 2 managers, and using both authentication and privacy for the third manager.

The configuration consists of three rows in the snmptargetAddrTable, and two rows in the snmpTargetTable.

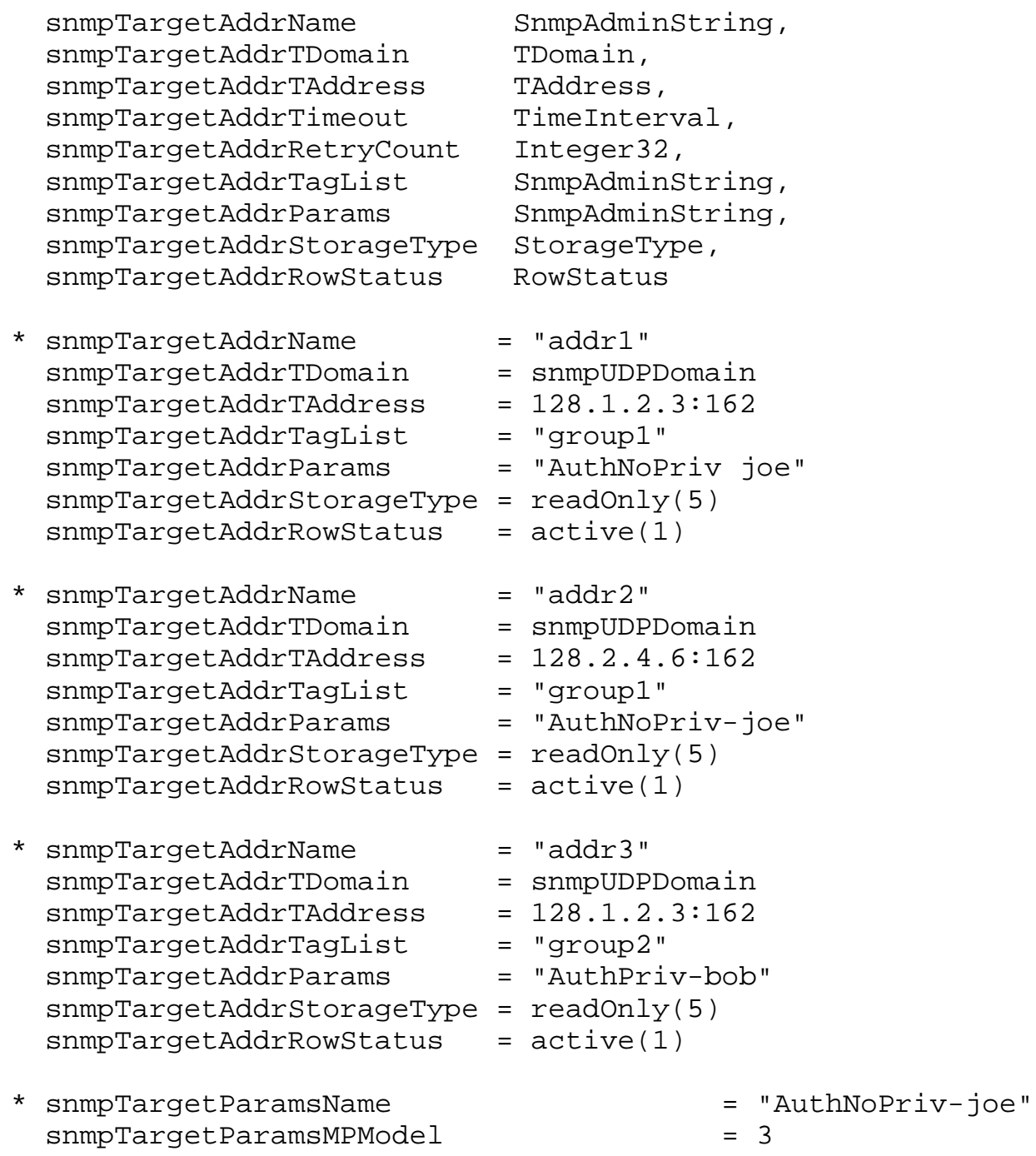




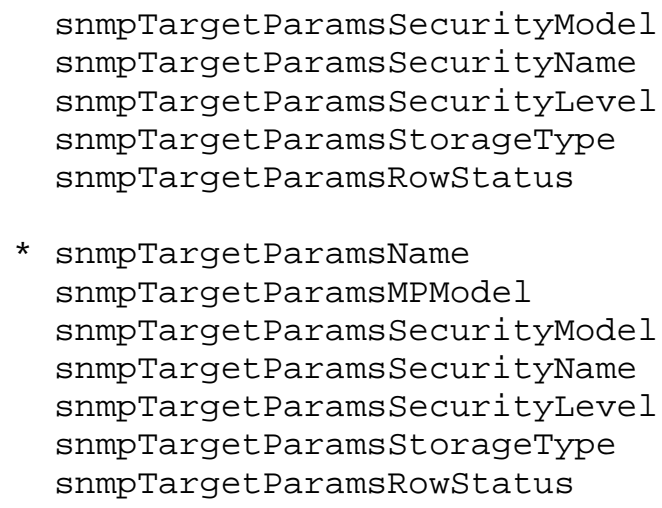

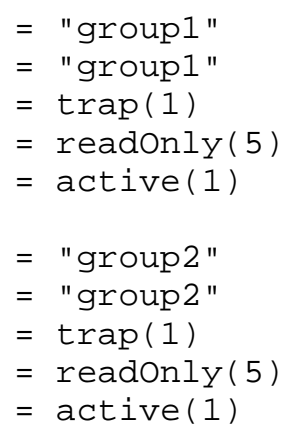

These entries define two groups of management targets. The first group contains two management targets:

$\begin{array}{rll} & \text { first target } & \text { second target } \\ & ---------- & ---------- \\ \text { messageProcessingModel } & \text { SNMPV3 } & \text { SNMPV3 } \\ \text { securityModel } & 3 \text { (USM) } & 3 \text { (USM) } \\ \text { securityName } & \text { "joe" } & \text { "joe" } \\ \text { securityLevel } & \text { authNoPriv(2) } & \text { authNoPriv(2) } \\ \text { transportDomain } & \text { snmpUDPDomain } & \text { snmpUDPDomain } \\ \text { transportAddress } & 128.1 .2 .3: 162 & 128.2 .4 .6: 162\end{array}$

And the second group contains a single management target:

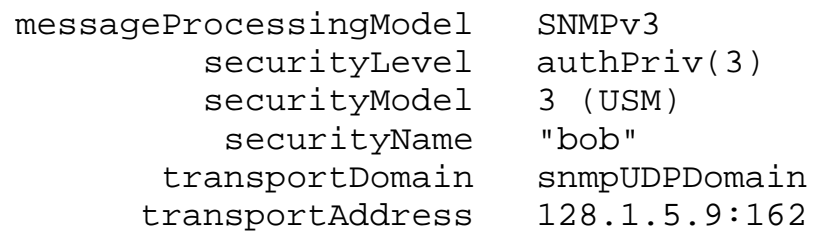


B. Full Copyright statement

Copyright (C) The Internet Society (1998). All Rights Reserved.

This document and translations of it may be copied and furnished to others, and derivative works that comment on or otherwise explain it or assist in its implementation may be prepared, copied, published and distributed, in whole or in part, without restriction of any kind, provided that the above copyright notice and this paragraph are included on all such copies and derivative works. However, this document itself may not be modified in any way, such as by removing the copyright notice or references to the Internet society or other Internet organizations, except as needed for the purpose of developing Internet standards in which case the procedures for copyrights defined in the Internet Standards process must be followed, or as required to translate it into languages other than English.

The limited permissions granted above are perpetual and will not be revoked by the Internet society or its successors or assigns.

This document and the information contained herein is provided on an "AS IS" basis and THE INTERNET SOCIETY AND THE INTERNET ENGINEERING TASK FORCE DISCLAIMS ALL WARRANTIES, EXPRESS OR IMPLIED, INCLUDING BUT NOT LIMITED TO ANY WARRANTY THAT THE USE OF THE INFORMATION HEREIN WILL NOT INFRINGE ANY RIGHTS OR ANY IMPLIED WARRANTIES OF MERCHANTABILITY OR FITNESS FOR A PARTICULAR PURPOSE. 